\title{
Possible Unification of Quantum Mechanics and General Relativity Theory Based on the Three-Dimensional Quantized Spaces
}

\author{
Jae - Kwang Hwang \\ JJJ Physics Laboratory, Brentwood, TN 37027, USA \\ jkhwang.koh@gmail.com
}

\begin{abstract}
Three-dimensional quantized space model is newly introduced. Quantum mechanics and relativity theory are explained in terms of the warped three-dimensional quantized spaces with the quantum time width $\left(\Delta \mathrm{t}=\mathrm{t}_{\mathrm{q}}\right)$. The energy is newly defined as the 4-dimensional space-time volume of $\mathrm{E}=$ $\mathrm{c} \Delta \mathrm{t} \Delta \mathrm{V}$ in the present work. It is shown that the wave function of the quantum mechanics is closely related to the warped quantized space shape with the space time-volume. The quantum entanglement and quantum wave function collapse are explained additionally. The special relativity theory is separated into the energy transition associated with the space-time shape transition of the matter and the momentum transition associated with the space-time location transition. Then, the quantum mechanics and the general relativity theory are about the 4dimensional space-time volume and the 4-dimensional space-time distance, respectively.
\end{abstract}

Keywords: Quantum mechanics; General and special relativity theories; Origins of the energy and mass; Space-time volume and distance; Origin of quantum wave function; Three-dimensional quantized spaces; Quantum entanglement; Space-time curvatures and quantum metrics

\section{Introduction}

Space and time are the mathematical concept and the energy is the physical concept of the space and time correlated status. The space with the non-zero energy can be called as the universe in the physics point of view. The universe has been so far discussed based on the unquantized space with the infinite number of dimensions. The physics and mathematics of the universe based on the standard model, quantum mechanics, general relativity, string theory etc. have been developed based on the unquantized space in Fig. 1. In the present work, the new concept of the n-dimensional quantized space $(n=1,2,3,4, \ldots)$ with the non-zero time width $(\Delta t)$ is, for the first time, introduced in Figs. 1 and 2 [1]. Four three-dimensional quantized spaces with total 12 dimensions are used to explain the universes including ours as shown in Figs. 1 and 2. Each n-dimensional quantized space has the energy when it has the time width if the energy is defined as $E_{n}=c \Delta t \Delta V_{n}$ in Table 1. $\Delta \mathrm{V}_{\mathrm{n}}$ is the volume of the $\mathrm{n}$-dimensional quantized space. For example, for the 3-dimensional quantized space, $\mathrm{E}=\mathrm{c} \Delta \mathrm{t} \Delta \mathrm{V}$ in Table 1 where $\Delta \mathrm{V}$ is the three-dimensional space volume in Fig. 3 [1]. Our universe is formed with the four 3-dimensional quantized spaces as shown in Figs. 1 and 2. Four 3-dimensional quantized spaces can be overlapped over the same states as shown in Fig. 1. There is the flat mother quantized space ( $\mathrm{x} 0 \mathrm{y} 0 \mathrm{z} 0$ space in Fig. 1) with the infinite time width and infinite space width. The mother quantized space cannot be warped because it has the infinite time width and infinite space width. The overlapped quantized spaces in Fig. 1 are associated with each other. The daughter quantized spaces (x1x2x3, x4x5x6 and $\times 7 \times 8 \times 9$ spaces in Fig. 1) with the finite time width and finite space width can be warped on the flat mother quantized space. 
Therefore, if one daughter quantized space is warped, other daughter quantized spaces are warped, too. The warped space is defined with the charge and rest mass in Fig. 2.

In the present work, the energy $\left(E_{n}\right)$ of the n-dimensional quantized flat space is defined as $E_{n}=$ $\mathrm{c} \Delta \mathrm{t} \Delta \mathrm{V}_{\mathrm{n}}$ where $\Delta \mathrm{V}_{\mathrm{n}}$ is the $\mathrm{n}$-dimensional quantized space volume and $\Delta \mathrm{t}$ is the time width [1]. The time momentum and the space momentum are not defined for the universe where $\Delta \mathrm{t}$ and $\Delta \mathrm{V}_{\mathrm{n}}$ have the infinite values. These infinite-energy $n$-dimensional quantized flat spaces are called as the $n-$ dimensional mother universes. If $\Delta t$ and $\Delta V_{n}$ have the finite values, the energy of $E_{n}$ is finite and the time momentum of $\mathrm{P}_{\mathrm{t}}=\mathrm{E}_{\mathrm{n}} / \mathrm{c}$ and the space momentum of $\mathrm{P}_{\mathrm{x}}$ have the finite values. These finite energy n-dimensional quantized universes are called as the n-dimensional daughter universes. Specifically, under the assumption that our universe is the 3-dimensional matter quantized space, the case of the 3-dimensional quantized spaces shown in Figs. 1 and 2 is explained in the present work.
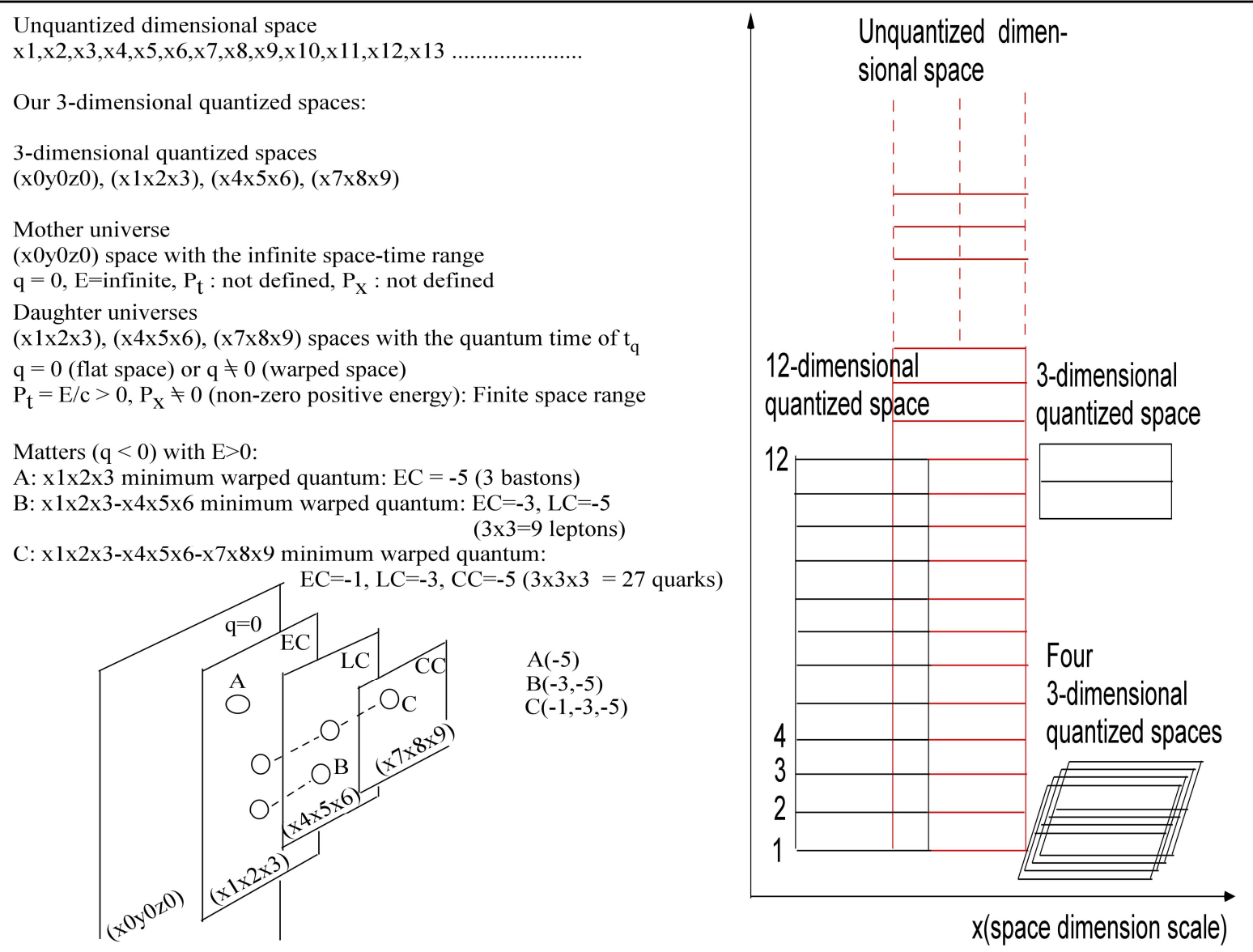

Fig. 1. 3-dimensional quantized spaces of $\mathrm{x} 0 \mathrm{y} 0 \mathrm{z} 0, \mathrm{x} 1 \mathrm{x} 2 \mathrm{x} 3, \mathrm{x} 4 \mathrm{x} 5 \mathrm{x} 6$ and $\mathrm{x} 7 \mathrm{x} 8 \mathrm{x} 9$ associated with each other $[11$. The unauantized snace is comnared with the auantized snaces.

Mathematically, all kinds of n-dimensional quantized flat spaces with $0<n \leq \infty$ including the space with the infinite number of the space dimensions exist in Fig. 1. In the present work, the mother universes of the $n=1$-, 2- and 3- dimensional quantized flat spaces with infinite energies are expressed as the $\mathrm{x} 0, \mathrm{x} 0 \mathrm{y} 0$ and $\mathrm{x} 0 \mathrm{y} 0 \mathrm{z} 0$, respectively. For example, the first 3-dimensional quantized space with the infinite space and time ranges (3-dimensional x0y0z0 mother universe)

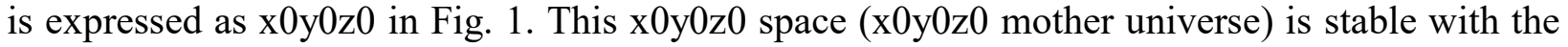


infinite energy in the physics point of view. In order for the space to have the physical meaning, the space should have the finite limited space and time ranges which can give the finite time and space momenta. These spaces with the finite energies can be warped in order to make the matters in Fig. 2. In the present work, those spaces with the physical meaning are expressed as the $x 1 \times 2 \times 3$, $\times 4 \times 5 \times 6$ and $\times 7 \times 8 \times 9$ spaces which are intertwined with each other in Fig. 1. Then the physical quantity of the energy $\left(\mathrm{E}=\mathrm{c} \Delta \mathrm{t} \Delta \mathrm{V}_{3}\right)$ in Table 1 can be defined for the three-dimensional quantized spaces such as the $\times 1 \times 2 \times 3, \times 4 \times 5 \times 6$ and $\times 7 \times 8 \times 9$ spaces. I think that a lot of three-dimensional quantized spaces (daughter universes) with the different sizes of the time width and space length can be created and annihilated, all the time, at the different time and different locations over the mother universe. These daughter universes will take place from the quantum fluctuations of the space-time which creates the energy.

\begin{tabular}{|c|c|c|c|}
\hline $\begin{array}{l}\text { Dimen- } \\
\text { sions } \\
\text { (n) }\end{array}$ & & Volume & $\begin{array}{l}\text { Distance } \\
(\Delta \ell)\end{array}$ \\
\hline 4 & Energy & $\begin{array}{l}E=c \Delta t \Delta V \\
=c \Delta t \Delta x 1 \Delta x 2 \Delta x 3 \\
=\int c t(x) d x \\
=\int_{\text {(Quantum mechanics) }} E|\Psi(x)|^{2} d x\end{array}$ & $\begin{array}{c}\Delta \ell^{2}=c^{2} \Delta t_{l}^{2}=c^{2} \Delta t^{2}+\Delta x 1^{2}+\Delta x 2^{2} \\
+\Delta x 3^{2}, \\
\Delta \ell^{2}=c^{2} \Delta t_{l}^{2}=c^{2} \Delta t^{2}+a\left(t_{l}\right)^{2} \\
\left(\Delta x 1^{2}+\Delta x 2^{2}+\Delta x 3^{2}\right) \\
=c^{2} \Delta t^{2}+a\left(t_{l}\right)^{2} \Delta x^{2} \\
\text { (Metric, General relativity) }\end{array}$ \\
\hline 3 & Volume & $V=\Delta x 1 \Delta x 2 \Delta x 3$ & $\begin{array}{c}\Delta \ell^{2}= \\
\Delta x 1^{2}+\Delta x 2^{2}+\Delta x 3^{2}\end{array}$ \\
\hline 2 & Area & $A=\Delta x 1 \Delta x 2$ & $\begin{array}{c}\Delta \ell^{2}= \\
\Delta x 1^{2}+\Delta x 2^{2}\end{array}$ \\
\hline 1 & Length & $l=\Delta x 1$ & $\Delta \ell^{2}=\Delta x 1^{2}$ \\
\hline
\end{tabular}

In the present work, the quantum length $\left(\mathrm{x}_{\mathrm{q}}\right)$ and quantum time $\left(\mathrm{t}_{\mathrm{q}}\right)$ with the relation of $\mathrm{t}_{\mathrm{q}}=\mathrm{x}_{\mathrm{q}} / \mathrm{c}$ are defined for the three-dimensional quantized spaces in Fig. 2. The quantum length is smaller than the Planck length $\left(\mathrm{x}_{\mathrm{p}}\right)$. If the matter with $\mathrm{E}>0$ is given, the time momentum $\left(\mathrm{P}_{\mathrm{t}}=\mathrm{E} / \mathrm{c}\right)$ is constant and positive. Therefore, the time of the matter is increasing constantly along the positive time axis. It is because all of the three dimensional quantized spaces are made up of the quantum spaces with the constant quantum space fluctuation time of $t_{q}=x_{q} / c$ as shown in Fig. 2. The quantum time of $\mathrm{t}_{\mathrm{q}}$ is the minimum size of the time. Also, the observable time increase of $\Delta \mathrm{t}_{1}=$ $\Delta \mathrm{x} / \mathrm{v}$ can be indirectly measured by the non-zero velocity of the matter. For example, $\Delta \mathrm{t}_{1}=\Delta \mathrm{x} / \mathrm{c}$ from the photon movement with the constant velocity of $c$. The space momentum of $\left(\mathrm{P}_{\mathrm{x}}=\mathrm{Ev} / \mathrm{c}^{2}\right)$ is changing according to the velocity of $\mathrm{v}$ and the space position of the matter can be increasing or decreasing depending on the direction of the velocity. Therefore, the time of the three-dimensional quantized spaces is originated from the constant fluctuation velocity $\left(\mathrm{c}=\mathrm{x}_{\mathrm{q}} / \mathrm{t}_{\mathrm{q}}\right)$ of the quantum spaces. Also, the mother universe has the infinite quantum time which means the stable mother universe because it takes the infinite time to fluctuate. It indicates that the mother universes are present permanently with the infinite quantum times and their daughter universes are fluctuating within the finite quantum times. Therefore, the quantum time fluctuation and time momentum are the origin of the time we on the earth have experienced. 
Physically only the three-dimensional quantized space is observed at the very short (instant) time width around us. We observe only the matters with the three-dimensional space volumes which

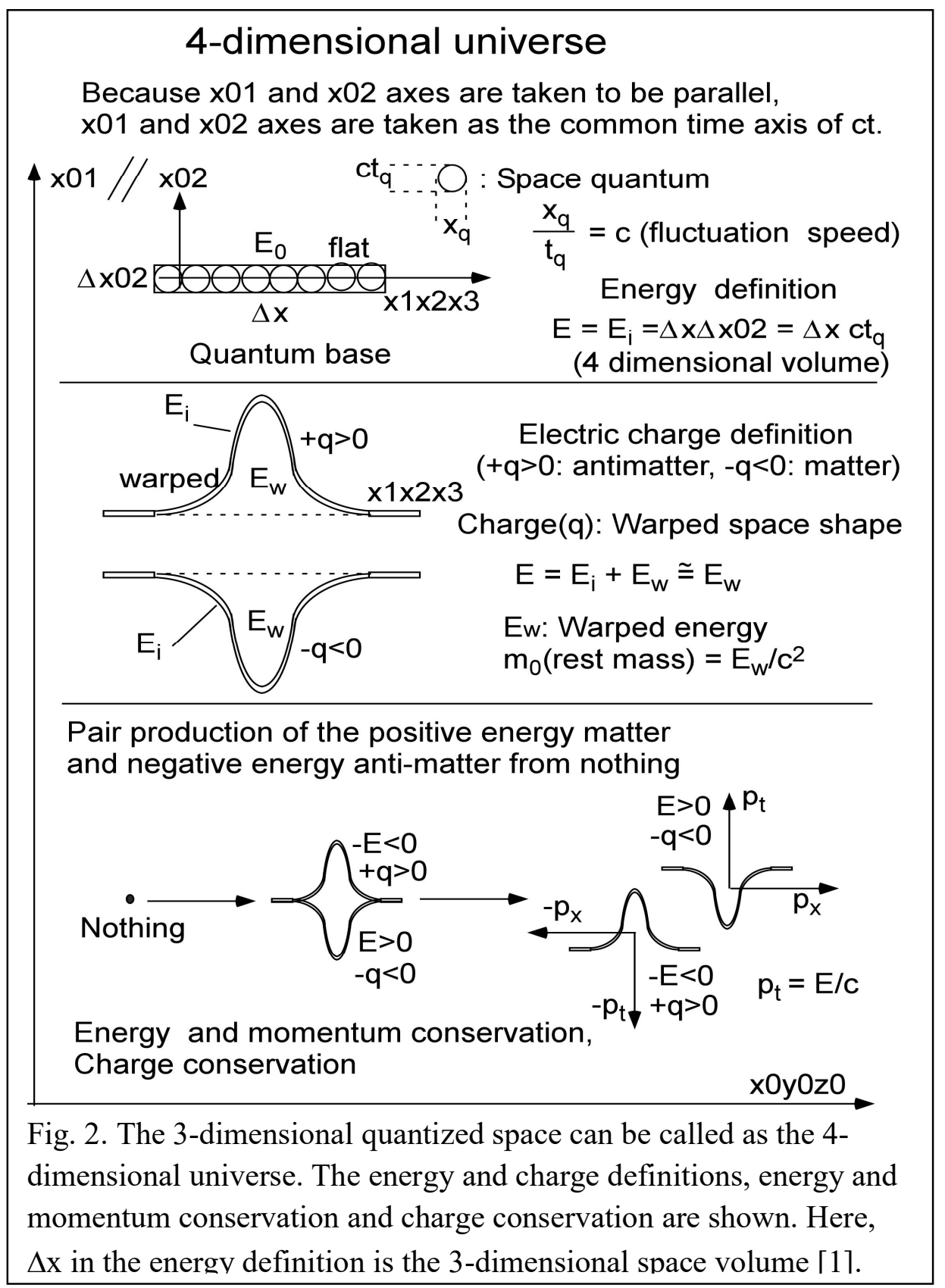

are made of the elementary particles with the electric charges of $2 / 3,0,-1 / 3$ and -1 . In the present work, it is assumed that we are living and locked in the three-dimensional quantized spaces of $\mathrm{x} 1 \times 2 \times 3, \mathrm{x} 4 \times 5 \times 6$ and $\times 7 \times 8 \times 9$ with the minimum time width of $\Delta \mathrm{t}=\mathrm{t}_{\mathrm{q}}$ (quantum time). In Fig. 2, if the $\mathrm{x} 1 \times 2 \times 3$ space is warped to the $+\mathrm{t}$ direction, it is called as the $+\mathrm{q}>0$ (positive electric charge) warping which creates the anti-matter universe. In Fig. 2, if the $x 1 \times 2 \times 3$ space is warped to the $-t$ direction, it is called as the $-\mathrm{q}<0$ (negative electric charge) warping which creates the matter universe. Electric (EC), lepton (LC) and color (CC) charges are defined to be the charges of the $\mathrm{x} 1 \times 2 \times 3, \times 4 \times 5 \times 6$ and $\times 7 \times 8 \times 9$ warped spaces, respectively, in the present work. The 3-dimensional quantized space can be, also, called as the 4-dimensional universe as shown in Fig. 2. Then, the 
time axis can be treated as the normal space axis in the 4-dimensional universe. Because the $\mathrm{x} 01$ and $\mathrm{x} 02$ axes are parallel to each other, the x01 and x02 axes in the 4-dimensional universe model can be considered as the common time axis of ct in the 3-dimensional quantized space model. The energy and charge definitions, energy and momentum conservation and charge conservation are shown for the summarization. Also, the pair production of the positive energy matter and negative
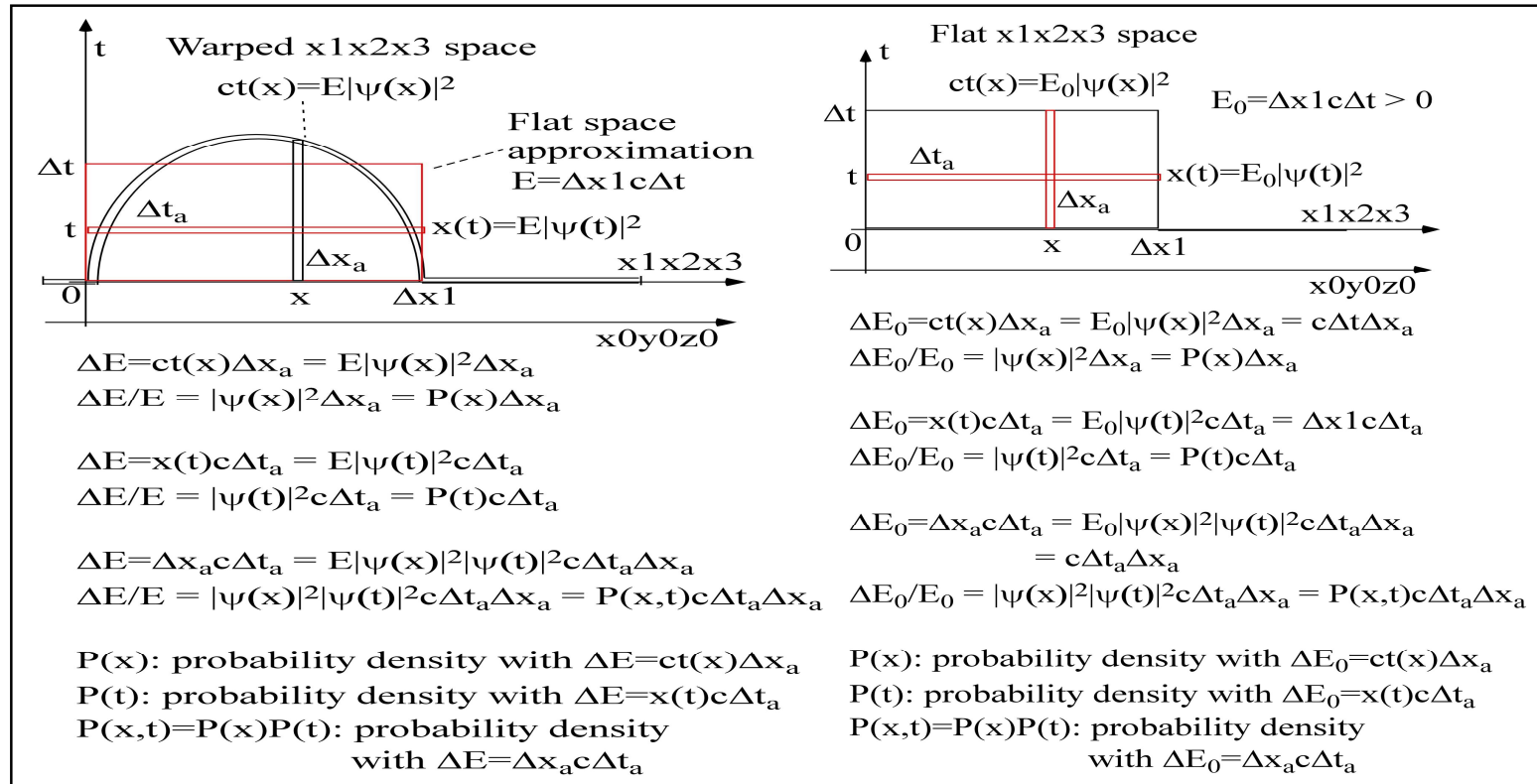

Fig. 3. $|\psi(\mathrm{x})|^{2},|\psi(\mathrm{t})|^{2}$ and $|\psi(\mathrm{x}, \mathrm{t})|^{2}=|\psi(\mathrm{x})|^{2}|\psi(\mathrm{x})|^{2}$ considered as the probability densities.

energy anti-matter from nothing is shown in Fig. 2. In the present work, quantum mechanics and relativity theory are explained in terms of the three-dimensional quantized space model as shown in Table $1 . \quad$ The space-time distance $\left(\Delta \ell^{2}=c^{2} \Delta t_{l}^{2}=c^{2} \Delta t^{2}+a\left(t_{l}\right)^{2}\left(\Delta x 1^{2}+\Delta x 2^{2}+\Delta x 3^{2}\right)\right)$ of the general relativity obtained under the condition of the energy conservation is the approximated distance of the real space-time distance $\left(\Delta \ell^{2}=c^{2} \Delta t_{l}^{2}=c^{2} \Delta t^{2}+\Delta x 1^{2}+\Delta x 2^{2}+\Delta x 3^{2}\right)$. The revised quantum mechanics and modified special and general relativity theories are discussed in terms of the three-dimensional quantized model. The quantum entanglement and quantum wave function collapse are explained additionally.

2. Quantum mechanics and modified relativity theory

\subsection{Revised quantum mechanics}

In quantum mechanics, Schrodinger equation is $E \psi=H \psi=\left(\frac{p^{2}}{2 m}+V\right) \psi$ and $E=\int E(x) d x=$ $\int E|\psi(x)|^{2} d x$. In the present work, $E=\int c t(x) d x$ in Figs. 2 and 3. Therefore, $\operatorname{ct}(\mathrm{x})=\mathrm{E}(\mathrm{x})=$ $\mathrm{E}|\psi(\mathrm{x})|^{2}$. Therefore, $|\psi(\mathrm{x})|^{2}=\operatorname{ct}(\mathrm{x}) / \mathrm{E}$ where $\mathrm{t}(\mathrm{x})$ is the time function of the warped space as shown in Fig. 3. It is the real meaning of the wave function introduced in the quantum mechanics. In Fig. 3 , it is shown that $|\psi(\mathrm{x})|^{2},|\psi(\mathrm{t})|^{2}$ and $|\psi(\mathrm{x}, \mathrm{t})|^{2}=|\psi(\mathrm{x})|^{2}|\psi(\mathrm{x})|^{2}$ are considered as the probability densities. The flat space can be explained by using the plane wave functions of $x$ and $t$ in 3 . The flat space means that the rest mass $\left(\mathrm{m}_{0}\right)$ is zero and it can be described only as the plane wave in Figs. 2 and 3. The flat $\mathrm{x} 1 \times 2 \times 3$ space with the time width of $\Delta t$ is shown in Figs. 3. In the present work, this flat space with the positive energy $\left(\mathrm{E}_{0}>0\right)$ is described by using the plane waves with 
the $\mathrm{p}_{\mathrm{t}}=\mathrm{E}_{0} / \mathrm{c}>0$ (along the $+\mathrm{t}$ direction) and $\mathrm{p}_{\mathrm{x}}>0$ (along $+\mathrm{x}$ direction) momenta. The plane wave functions are $\psi(x)=A e^{\frac{i}{\hbar} P_{x} x}$ and $\psi(t)=B e^{\frac{i E_{0}}{c} c t}$. Then, $\mathrm{p}_{\mathrm{x}}=-i \hbar \frac{d}{d x}$ and $\mathrm{p}_{\mathrm{t}}=\mathrm{E}_{0} / \mathrm{c}=-\mathrm{i} \hbar \frac{d}{c d t}$. From these equations, $\mathrm{E}_{0}=\mathrm{c} \mathrm{p}_{\mathrm{t}}=-\mathrm{i} \hbar \frac{d}{d t}$ and $\Psi(\mathrm{x}, \mathrm{t})=\psi(\mathrm{x}) \psi(\mathrm{t})$. And $\mathrm{p}_{\mathrm{x}}=\hbar \mathrm{k}_{\mathrm{x}}$ and $\mathrm{E}_{0}=\mathrm{cp}_{\mathrm{t}}=\hbar \omega$. Note that $\psi(t)=B e^{-\frac{i}{\hbar} E t}$ and $\mathrm{E}=\mathrm{i} \frac{d}{d t}$ which have been given and used in the well-known quantum

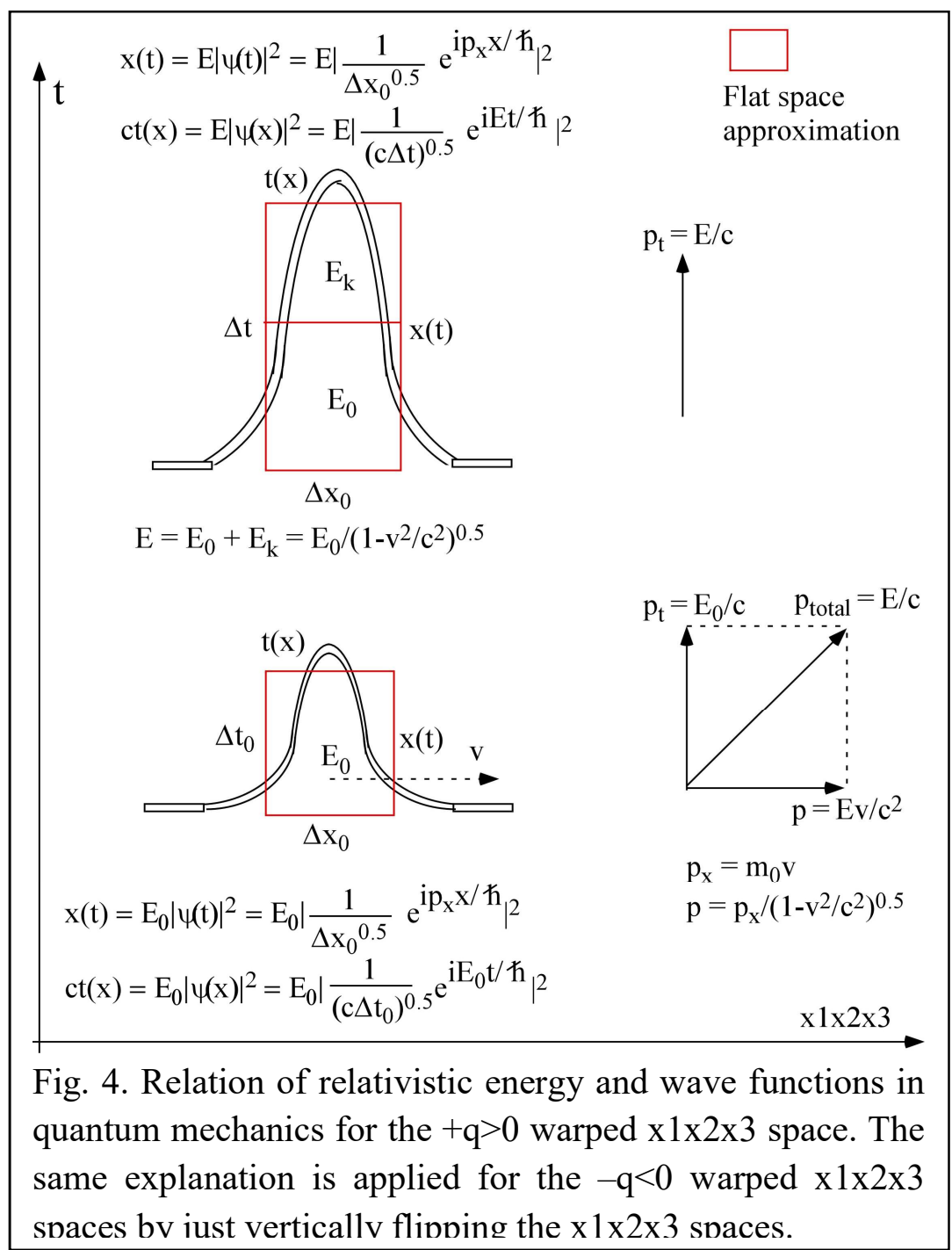

mechanics are different in the sign from those given from the present study. $p_{x}$ is the nonrelativistic operator because the plane wave does not warp the $\mathrm{x} 1 \mathrm{x} 2 \mathrm{x} 3$ space. The flat space is not changed by the space momentum of the space plane wave. Therefore, the flat space energy is $\mathrm{E}_{0}=$ $\Delta \mathrm{xc} \Delta \mathrm{t}=\Delta \mathrm{x} 1 \mathrm{c} \Delta \mathrm{t}$ as shown in Fig. 3 .

Then, $\mathrm{p}_{\text {total }}{ }^{2}=\mathrm{E}^{2} / \mathrm{c}^{2}=\mathrm{p}_{\mathrm{x}}{ }^{2}+\mathrm{E}_{0}{ }^{2} / \mathrm{c}^{2}, \overrightarrow{p_{\text {total }}}=p_{x} \hat{\imath}+p_{t} \hat{t}, \overrightarrow{x_{\text {total }}}=x \hat{\imath}+c t \hat{t}$, and $\mathrm{E}_{0}=\Delta \mathrm{xc} \Delta \mathrm{t}$. Then $\psi(x, t)=A B e^{\frac{i}{\hbar} \overrightarrow{p_{\text {total }}} \cdot \overrightarrow{x_{\text {total }}}}=A e^{\frac{i}{\hbar} P_{x} x} B e^{\frac{i E_{0}}{\hbar c} c t}$, and $d E=c d x d t=c d x_{a} d t_{a}$.

$\iint|\psi(x, t)|^{2} d x c d t=A^{2} B^{2} \Delta x c \Delta t=1$. Then $\mathrm{A}^{2}=1 / \Delta \mathrm{x}$ and $\mathrm{B}^{2}=1 /(\mathrm{c} \Delta \mathrm{t})$. Therefore, $\mathrm{A}^{2} \mathrm{~B}^{2}=$ $1 /(\Delta \mathrm{xc} \Delta \mathrm{t})$, and $\psi(x, t)=\frac{1}{\sqrt{\Delta x c \Delta t}} e^{\frac{i}{\hbar} P_{x} x} e^{\frac{i E_{0}}{\hbar c} c t}$.

For the 3-dimensional flat space, 


$$
\psi(x, t)=\frac{1}{\sqrt{\Delta x_{1} \Delta x_{2} \Delta x_{3} c \Delta t}} e^{\frac{i}{\bar{\hbar}} \overrightarrow{p_{x}} \cdot \vec{x}} e^{\frac{i E_{0}}{\hbar c} c t}, \vec{x}=x_{1} \hat{\imath}+x_{2} \hat{\jmath}+x_{3} \hat{k} .
$$

The same procedure can be applied for the $\mathrm{x} 4 \times 5 \times 6$ and $\times 7 \times 8 \times 9$ flat spaces and this explanation can be extended easily to the $\mathrm{x} 1 \times 2 \times 3-\mathrm{x} 4 \times 5 \times 6$ and $\mathrm{x} 1 \times 2 \times 3-\mathrm{x} 4 \times 5 \times 6-\mathrm{x} 7 \mathrm{x} 8 \times 9$ flat spaces.

The flat one-dimensional space with the energy of $E_{0}$ is shown in Figs. 2, 3 and 4. Then, $\mathrm{p}_{\text {total }}{ }^{2}=\mathrm{E}^{2} / \mathrm{c}^{2}=\mathrm{p}_{\mathrm{x}}{ }^{2}+\mathrm{E}_{0}{ }^{2} / \mathrm{c}^{2}$ and $\mathrm{E}_{0}=\Delta \mathrm{x} 1 \mathrm{c} \Delta \mathrm{t}$. The flat space is treated non-relativistically because it does not warp the space while it is moving with the light speed of $\mathrm{v}=\mathrm{c}$. The flat space with the energy of $\mathrm{E}_{0}$ should be described by using the plane space and time wave functions of $\psi(x)=$ $\frac{1}{\sqrt{\Delta x 1}} e^{\frac{i}{\hbar} P_{x} x}$ and $\psi(t)=\frac{1}{\sqrt{c \Delta t}} e^{\frac{i E_{0}}{\hbar} c t}$. Then, $\mathrm{p}_{\mathrm{x}}=\mathrm{E}_{0} \mathrm{v} / \mathrm{c}^{2}=\mathrm{E}_{0} / \mathrm{c}=\mathrm{p}_{\mathrm{t}}$ because $\mathrm{v}$ is equal to $\mathrm{c}$ for the plane waves. And from $p_{\mathrm{x}}=\hbar \mathrm{k}_{\mathrm{x}}$ and $\mathrm{E}_{0}=\mathrm{cp}_{\mathrm{t}}=\hbar \omega_{0}, \mathrm{k}_{\mathrm{x}}=\omega_{0} / \mathrm{c}$ is obtained. And for the massless photon, $\mathrm{E}^{2}=2 \mathrm{E}_{0}^{2}$. Because $\mathrm{p}_{\mathrm{x}}=-\mathrm{i} \hbar \frac{d}{d x}$ and $\mathrm{p}_{\mathrm{t}}=\mathrm{E}_{0} / \mathrm{c}=-\mathrm{i} \hbar \frac{d}{c d t},-\mathrm{i} \hbar \frac{d}{d x} \psi(\mathrm{x}) \psi(\mathrm{t})=-\mathrm{i} \hbar \frac{d}{c d t} \psi(\mathrm{x}) \psi(\mathrm{t})$. Therefore, $\frac{d}{d x} \psi(\mathrm{x}) \psi(\mathrm{t})=\frac{d}{c d t} \psi(\mathrm{x}) \psi(\mathrm{t})$ from $\mathrm{p}_{\mathrm{x}}=\mathrm{E}_{0} / \mathrm{c}$. And from $\mathrm{p}_{\mathrm{x}}{ }^{2}=\mathrm{E}_{0}^{2} / \mathrm{c}^{2}, \frac{d^{2}}{d x^{2}} \psi(\mathrm{x}) \psi(\mathrm{t})=$ $\frac{d^{2}}{c^{2} d t^{2}} \psi(\mathrm{x}) \psi(\mathrm{t})$. This is the well-known wave equation with the wave velocity of $\mathrm{v}=\mathrm{c}$. This can be easily extended to the flat three-dimensional space with the energy of $\mathrm{E}_{0}$.

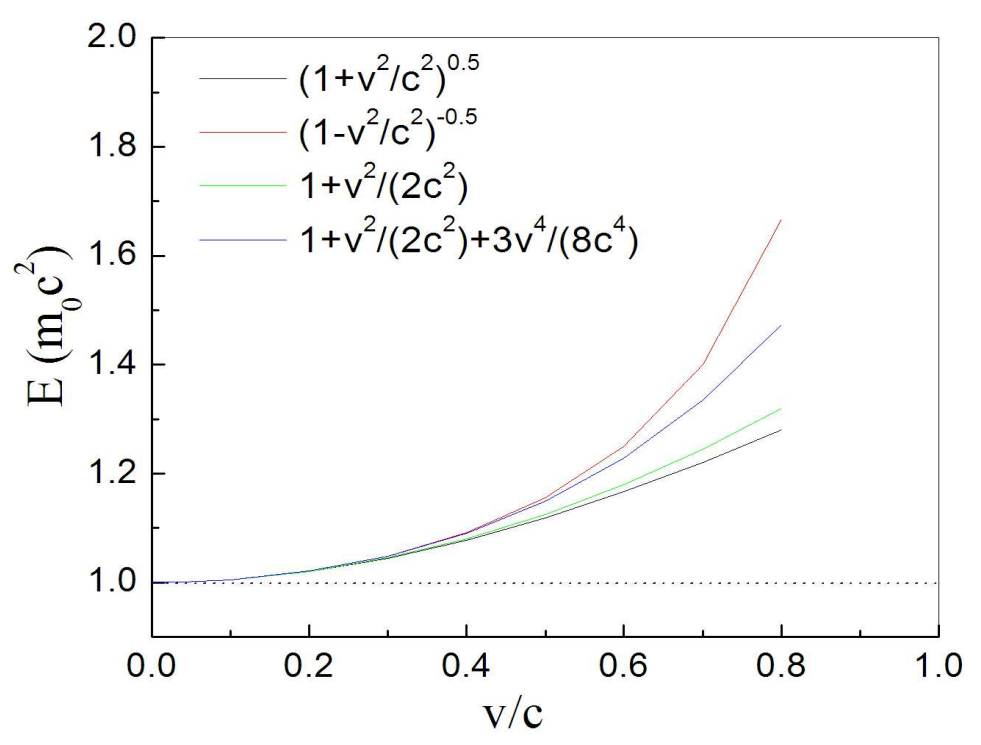

Fig. 5. Comparison of the total energies (E) including the kinetic energies $\left(\mathrm{E}_{\mathrm{k}}\right)$.

The warped $\mathrm{x} 1 \times 2 \times 3$ space can be described by using the wave functions in Fig. 4 . If the warped space is more warped by the space momentum of the warped matter. it should be treated relativistically. Therefore, the modified warped space has the energy of $E=\Delta x_{0} c \Delta t$ increased by the kinetic energy from the rest mass energy of $\mathrm{E}_{0}=\Delta \mathrm{x}_{0} \mathrm{c} \Delta \mathrm{t}_{0}$ as shown in Figs. 4. In this case, $\mathrm{E}$ is positive for the flat space approximation of the warped space. Relativistically, $\psi(x)=$ $A(x)$ and $\psi(t)=B(t)$ can be used to derive the space and time wave functions of the warped space in Fig. 4. $p_{x}^{\prime}$ is zero because of the relativistic effect in Fig. 4. A(x) and B(t) can warp the space as the functions of $x$ and $t$. The time wave function of $\psi(t)$ for the warped space is hard to be directly calculated. Therefore, $\psi(t)$ is indirectly calculated from the $x 1 \times 2 \times 3$ flat space approximation of the warped space as seen as a red squares with the energy of $E$ in Fig. 4. This means that the plane wave function of $\psi(t)$ is used as the approximated form of the actual $\psi(t)$ 
wave function. Because the time wave function is obtained from the flat space approximation with the energy of $\mathrm{E}$ in Fig. 4, a time wave function is taken as the plane wave function of $\psi(t)=$ $\frac{1}{\sqrt{c \Delta t}} e^{\frac{i E}{\hbar c} c t}$. And because the space momentum of $p_{x}^{\prime}$ is zero for the relativistic effect, the space wave function is $\psi(x)=A(x)$. A real space wave function of $\psi(\mathrm{x})$ in Fig.4 can be obtained from the Schrodinger equation of $E \psi(x)=\mathrm{E}_{0} /\left(1-\mathrm{v}^{2} / \mathrm{c}^{2}\right)^{0.5} \psi(x)$ as follows. In the present work, onedimensional Schrodinger equation with $\Delta \mathrm{E}=\operatorname{ct}(\mathrm{x}) \Delta \mathrm{x}_{\mathrm{a}}=\mathrm{E}|\psi(\mathrm{x})|^{2} \Delta \mathrm{x}_{\mathrm{a}}$ along the $\mathrm{x} 1$ axis in Figs. 3 and 4 have been shown for the simplicity.

The warped space case with the non-zero rest mass $\left(\mathrm{m}_{0}\right)$ moving with the velocity of $\mathrm{v}$ within the $\mathrm{x} 1 \times 2 \times 3$ space is shown in Fig. 4. This warped space means that it has the non-zero rest mass $\left(\mathrm{m}_{0}\right)$ in Fig. 3. When the rest mass $\left(\mathrm{m}_{0}\right)$ moves with $\mathrm{v}$ in the flat $\mathrm{x} 1 \mathrm{x} 2 \mathrm{x} 3$ space, it warps the $\mathrm{x} 1 \mathrm{x} 2 \mathrm{x} 3$ space as shown in Fig. 4. It is the relativistic effect. This additional warped space energy is the same as the kinetic energy $\left(\mathrm{E}_{\mathrm{k}}\right)$. The energy of the matter moving with the velocity of $\mathrm{v}$ within the $\mathrm{x} 1 \mathrm{x} 2 \mathrm{x} 3$ space is $\mathrm{E}=\mathrm{E}_{0} /\left(1-\mathrm{v}^{2} / \mathrm{c}^{2}\right)^{0.5}$ in Fig. 4. Here, $\mathrm{E}_{0}=\mathrm{m}_{0} \mathrm{c}^{2} . E \psi(x) \psi(t)=\mathrm{E}_{0} /\left(1-\mathrm{v}^{2} / \mathrm{c}^{2}\right)^{0.5} \psi(x) \psi(t)$ for the warped space. Then, $E \approx m_{0} c^{2}\left(1+\frac{v^{2}}{2 c^{2}}+\frac{3 v^{4}}{8 c^{4}}\right)=m_{0} c^{2}+\frac{p_{x}^{2}}{2 m_{0}}+\frac{3 p_{x}^{4}}{8 m_{0}^{3} c^{2}}$ where $\mathrm{p}_{\mathrm{x}}=\mathrm{m}_{0 \mathrm{~V}}$ and $\mathrm{E}=\mathrm{E}_{0}+\mathrm{E}_{\mathrm{k}}$. Therefore, $E_{k} \approx \frac{p_{x}^{2}}{2 m_{0}}$. From the equation of $\mathrm{E}=\mathrm{E}_{\mathrm{k}}+\mathrm{E}_{0}+\mathrm{V}(\mathrm{x})$ with the additional potential energy of $\mathrm{V}(\mathrm{x}), \psi(\mathrm{x})$ can be calculated by using the Schrodinger equation of $E \psi(x)=$ $H \psi(x)=\left(E_{0}+\frac{p_{x}^{2}}{2 m}+V(x)\right) \psi(x) . \quad$ And, $p_{x}=-i \hbar \frac{d}{d x}$ and $E=\int E(x) d x=\int E|\psi(x)|^{2} d x$. From $\mathrm{E} \psi(\mathrm{t})=-\mathrm{i} \hbar \frac{d}{d t} \psi(\mathrm{t})$ and $\psi(t)=\frac{1}{\sqrt{c \Delta t}} e^{\frac{i E}{\hbar c} c t}$ obtained by the flat space approximation, the wave function of the Schrodinger equation can be expressed as $\psi(x, t)=\psi(x) \frac{1}{\sqrt{c \Delta t}} e^{\frac{i E}{\hbar c} c t}$. The same discussion can be applied for the particle moving in the $\mathrm{x} 1 \mathrm{x} 2 \mathrm{x} 3$ space as shown in Fig. 4 . The relativistic space momentum is $\mathrm{p}_{\mathrm{x}}($ relativistic $)=\mathrm{p}_{\mathrm{x}} /\left(1-\mathrm{v}^{2} / \mathrm{c}^{2}\right)^{0.5}=\frac{1}{\sqrt{1-\frac{v^{2}}{c^{2}}}}(-i \hbar) \frac{d}{d x}$.

For the high velocity case, it is thought that $\mathrm{E}_{\mathrm{k}} \approx \frac{p_{x}^{2}}{2 m_{0}}+\frac{3 p_{x}^{4}}{8 m_{0}^{3} c^{2}}$ gives the better prediction than $\mathrm{E}_{\mathrm{k}}$ $\approx \frac{p_{x}^{2}}{2 m_{0}}$ as shown in Fig. 5 . Therefore, for the high velocity case, the revised Schrodinger equation of $E \psi(x)=H \psi(x)=\left(E_{0}+\frac{p_{x}^{2}}{2 m}+\frac{3 p_{x}^{4}}{8 m_{0}^{3} c^{2}}+V(x)\right) \psi(x)$ can be used to calculate the wave function of $\psi(\mathrm{x})$ rather than the Schrodinger equation of $E \psi(x)=H \psi(x)=\left(E_{0}+\frac{p_{x}^{2}}{2 m}+\right.$ $V(x)) \psi(x)$. Also, $\psi_{\mathrm{s}}(\mathrm{x})$ is calculated from the well-known Schrodinger equation of $E_{s} \psi_{s}(x)=$ $\left(E-E_{0}\right) \psi_{s}(x)=H \psi_{s}(x)=\left(\frac{p_{x}^{2}}{2 m}+V(x)\right) \psi_{s}(x)$. Then, $\mathrm{E}_{\mathrm{s}} \psi_{\mathrm{s}}(\mathrm{t})=-\mathrm{i \hbar} \frac{d}{d t} \psi_{\mathrm{s}}(\mathrm{t})$ and $\psi_{s}(t)=$ $\frac{1}{\sqrt{c \Delta t_{s}}} e^{\frac{i E_{s}}{\hbar c} c t}$. Because $\mathrm{E}_{0}$ is the constant energy of $\mathrm{m}_{0} \mathrm{c}^{2}$ in Fig. 4,

$$
\psi(t)=\frac{1}{\sqrt{c \Delta t}} e^{i \frac{i E}{\hbar c} c t}=\frac{1}{\sqrt{c b \Delta t_{s}}} e^{\frac{i\left(E_{s}+E_{0}\right)}{\hbar} c t}=\frac{1}{\sqrt{b}} e^{\frac{i E_{0}}{\hbar c} c t} \frac{1}{\sqrt{c \Delta t_{s}}} e^{\frac{i E_{s}}{\hbar c} c t}=\frac{1}{\sqrt{b}} e^{\frac{i E_{0}}{\hbar c} c t} \psi_{s}(t)
$$


And, generally, $\psi(x)=\psi_{s}(x)$ because $\mathrm{E}_{0}$ (rest energy) can be taken as the reference energy for the whole space $(\mathrm{x})$ region of interest. In other words, only $\Delta \mathrm{t}_{\mathrm{s}}$ is increased to $\Delta \mathrm{t}=\mathrm{b} \Delta \mathrm{t}_{\mathrm{s}}$ but $\Delta \mathrm{x}$ is the same as $\Delta \mathrm{x}_{\mathrm{s}}$ on the flat space approximation by adding the constant energy of $\mathrm{E}_{0}$ in Fig. 4 . $\mathrm{E}=$ $\mathrm{c} \Delta \mathrm{t} \Delta \mathrm{x}=\mathrm{cb} \Delta \mathrm{t}_{\mathrm{s}} \Delta \mathrm{x}$ and $\mathrm{E}_{\mathrm{s}}=\mathrm{c} \Delta \mathrm{t}_{\mathrm{s}} \Delta \mathrm{x}$. And $\mathrm{b}=\mathrm{E} / \mathrm{E}_{\mathrm{s}}=\mathrm{E} /\left(\mathrm{E}-\mathrm{E}_{0}\right)$. Then, $\operatorname{ct}(\mathrm{x})=\mathrm{E} \mid \psi\left(\left.\mathrm{x}\right|^{2}\right.$ and $\operatorname{ct}_{\mathrm{s}}(\mathrm{x})=$ $\mathrm{E}_{\mathrm{s}}\left|\psi_{\mathrm{s}}(\mathrm{x})\right|^{2}=\mathrm{E}_{\mathrm{s}}|\psi(\mathrm{x})|^{2}$. Therefore, the well-known Schrodinger equation of $E_{s} \psi_{s}(x)=(E-$ $\left.E_{0}\right) \psi_{s}(x)=H \psi_{s}(x)=\left(\frac{p_{x}^{2}}{2 m}+V(x)\right) \psi_{s}(x)$ has been used instead of the Schrodinger equation of $E \psi(x)=H \psi(x)=\left(E_{0}+\frac{p_{x}^{2}}{2 m}+V(x)\right) \psi(x)$.

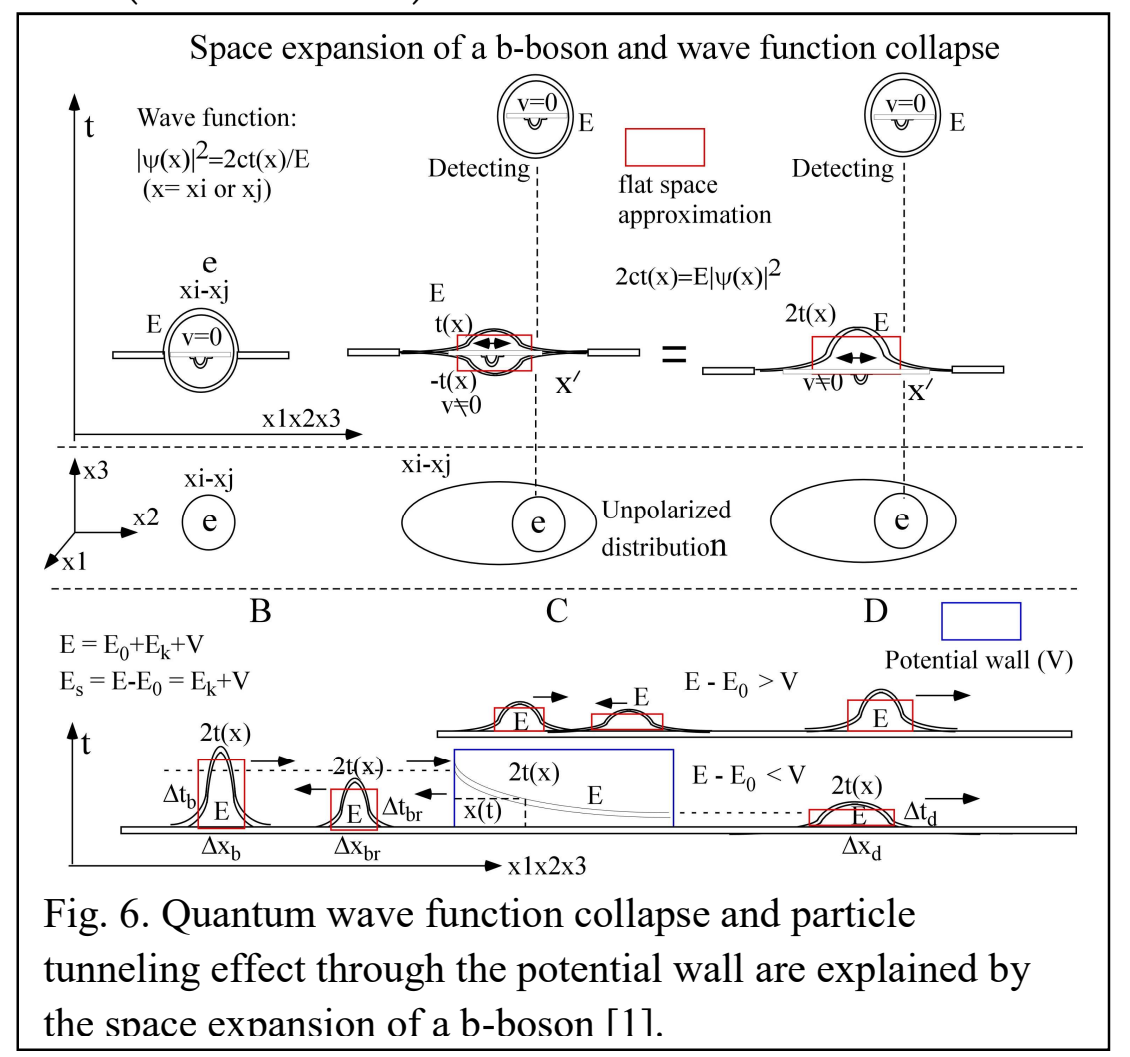

In other words, because $\mathrm{E}_{0}$ is added as the constant energy for the whole space $(\mathrm{x})$ region where the particle with the rest energy $\mathrm{E}_{0}$ moves, $\mathrm{E}_{0}$ can be taken as the reference energy in Fig. 4 . It indicates that when it is based on the reference energy of $E_{0}$, the well-known Schrodinger equation of

$E_{s} \psi_{s}(x)=\left(E-E_{0}\right) \psi_{s}(x)=H \psi_{s}(x)=\left(\frac{p_{x}^{2}}{2 m}+V(x)\right) \psi_{s}(x)$ can be used instead of the original Schrodinger equation of $E \psi(x)=H \psi(x)=\left(E_{0}+\frac{p_{x}^{2}}{2 m}+V(x)\right) \psi(x)$. And based on the reference energy of $E_{0}, E_{s} \psi_{s}(\mathrm{t})=-i \hbar \frac{d}{d t} \psi_{s}(\mathrm{t})$ and $\psi_{s}(t)=\frac{1}{\sqrt{c \Delta t_{s}}} e^{\frac{i E_{s}}{\hbar c} c t}$ can be used instead of the original equations of $\mathrm{E} \psi(\mathrm{t})=-i \hbar \frac{d}{d t} \psi(\mathrm{t})$ and $\psi(t)=\frac{1}{\sqrt{c \Delta t}} e^{\frac{i E}{\hbar c} c t}$ obtained by the flat space approximation. Then , $\psi(x)=\psi_{s}(x)$ and 
$\psi(t)=\frac{1}{\sqrt{c \Delta t}} e^{\frac{i E}{\hbar c} c t}=\frac{\sqrt{E-E_{0}}}{\sqrt{E}} e^{\frac{i E_{0}}{\hbar c} c t} \psi_{s}(t)$.

Now, as one example, I am going to discuss the particle like an electron moving to the $+x$ direction on the flat $\mathrm{x} 1 \times 2 \times 3$ space free of the potential wall as shown in the region B of Fig. 6 . Then the free electron can be described as the wave function with the relations of $2 \operatorname{ct}(\mathrm{x})=\mathrm{E}|\psi(\mathrm{x}, \mathrm{t})|^{2}$. In the flat space approximation of the red box in Figs. 4 and 6, the plane space and time wave functions of $\psi(x)=\frac{1}{\sqrt{\Delta x_{b}}} e^{\frac{i}{\hbar} P x_{x} x}=\frac{1}{\sqrt{\Delta x_{b}}} e^{\frac{i}{\hbar} \sqrt{2 m\left(E-E_{0}\right)} x}$ and $\psi(t)=\frac{1}{\sqrt{c \Delta t_{b}}} e^{\frac{i E}{\hbar c} c t}$ can be used for an electron moving to the $+\mathrm{x}$ direction toward the potential wall. Here, $\mathrm{E}=\mathrm{c} \Delta \mathrm{t}_{\mathrm{b}} \Delta \mathrm{x}_{\mathrm{b}}$. The space wave function of $\psi(x)=\frac{1}{\sqrt{\Delta x_{b}}} e^{\frac{i}{\hbar} P_{x} x}$ is the solution of $E \psi(x)=H \psi(x)=\left(E_{0}+\frac{p_{x}^{2}}{2 m}\right) \psi(x)$ and (E$\left.E_{0}\right) \psi(x)=H \psi(x)=\left(\frac{p_{x}^{2}}{2 m}\right) \psi(x)$. The general solution considering the $+\mathrm{x}$ and $-\mathrm{x}$ moving directions of the free electron in this region is $\psi(x)=\frac{F}{\sqrt{\Delta x_{b}}} e^{\frac{i}{\hbar} P_{x} x}+\frac{G}{\sqrt{\Delta x_{b r}}} e^{-\frac{i}{\hbar} P_{x} x}$ in Fig. 6. F and $\mathrm{G}$ are the coupling constants and $E_{s}=\left(E-E_{0}\right)=\left(\frac{p_{x}^{2}}{2 m}\right)$.

When an electron meets the potential wall with $\mathrm{E}_{\mathrm{s}}<\mathrm{V}$ (constant) in the region $\mathrm{C}$ of Fig. 6, the space wave function of the electron b-boson [1] has the tail into the potential wall. The space wave function of this tail part can be deduced from the Schrodinger equation of $\left(E-E_{0}\right) \psi(x)=$ $H \psi(x)=\left(\frac{p_{x}^{2}}{2 m}+V\right) \psi(x)$ by assuming the negative kinetic energy of $\mathrm{E}_{\mathrm{k}}=-\left(\mathrm{V}-\left(\mathrm{E}_{-} \mathrm{E}_{0}\right)\right)<0$ with the imaginary space momentum of $\mathrm{p}_{\mathrm{x}}=\mathrm{i} \sqrt{2 m\left(V-\left(E-E_{0}\right)\right)}$ under the temporary reference energy condition of $\mathrm{V}$. The positive kinetic energy stands for the free electron with that kinetic energy and the negative kinetic energy indicates the bound electron within the potential wall. The space probability density of the bound electron b-boson [1] deformed to the tail form is shown as $2 t(x)$ in Fig. 6. The space and time of this electron within the potential wall cannot be approximated by the flat space because those have the decaying wave function of $\psi(x)=A e^{-\sqrt{\frac{2 m\left(V-\left(E-E_{0}\right)\right)}{\hbar^{2}}} x}$ and $\psi(t)$. Within the potential wall with the constant potential energy of $\mathrm{V}>\mathrm{E}-\mathrm{E}_{0}$, the space wave function of $\psi(x)=A e^{-\sqrt{\frac{2 m\left(V-\left(E-E_{0}\right)\right)}{\hbar^{2}} x}}$ is the solution of $\left(E-E_{0}\right) \psi(x)=H \psi(x)=\left(\frac{p_{x}^{2}}{2 m}+\right.$ $V) \psi(x)$. The space wave function of $\psi(x)=A e^{-\sqrt{\frac{2 m\left(\left(V+E_{0}\right)-E\right)}{\hbar^{2}}} x}$ is the solution of $E \psi(x)=$ $H \psi(x)=\left(\frac{p_{x}^{2}}{2 m}+\left(V+E_{0}\right)\right) \psi(x)$, too. The free electron after going through the potential wall has the plane space and time wave functions of $\psi(x)=\frac{1}{\sqrt{\Delta x_{d}}} e^{\frac{i}{\hbar} P_{x} x}$ and $\psi(t)=\frac{1}{\sqrt{c \Delta t_{d}}} e^{\frac{i E}{\hbar c} c t}$ from the flat space approximation of the red box in Figs. 4 and 6. The coupling constant A, F and G can be calculated from the boundary conditions. When an electron moves to the $+x$ direction on the $\mathrm{x} 1 \mathrm{x} 2 \mathrm{x} 3$ flat space out of the potential wall in Fig. 6, the time width is decreasing from $\Delta \mathrm{t}_{\mathrm{b}}$ to $\Delta \mathrm{t}_{\mathrm{d}}$ and the space width is increasing from $\Delta \mathrm{x}_{\mathrm{b}}$ to $\Delta \mathrm{x}_{\mathrm{d}}$. The total energy is expressed by the relativistic equation of $\mathrm{E}=\mathrm{E}_{0} /\left(1-\mathrm{v}^{2} / \mathrm{c}^{2}\right)^{0.5}$. The relativistic space momentum can be obtained by the equation of $\mathrm{p}_{\mathrm{x}}($ relativistic $)=\mathrm{p}_{\mathrm{x}} /\left(1-\mathrm{v}^{2} / \mathrm{c}^{2}\right)^{0.5}=\frac{1}{\sqrt{1-\frac{v^{2}}{c^{2}}}}(-i \hbar) \frac{d}{d x}$. 
When an electron meets the potential wall with $E_{s}>V$ (constant) in the region $C$ of Fig. 6 , the

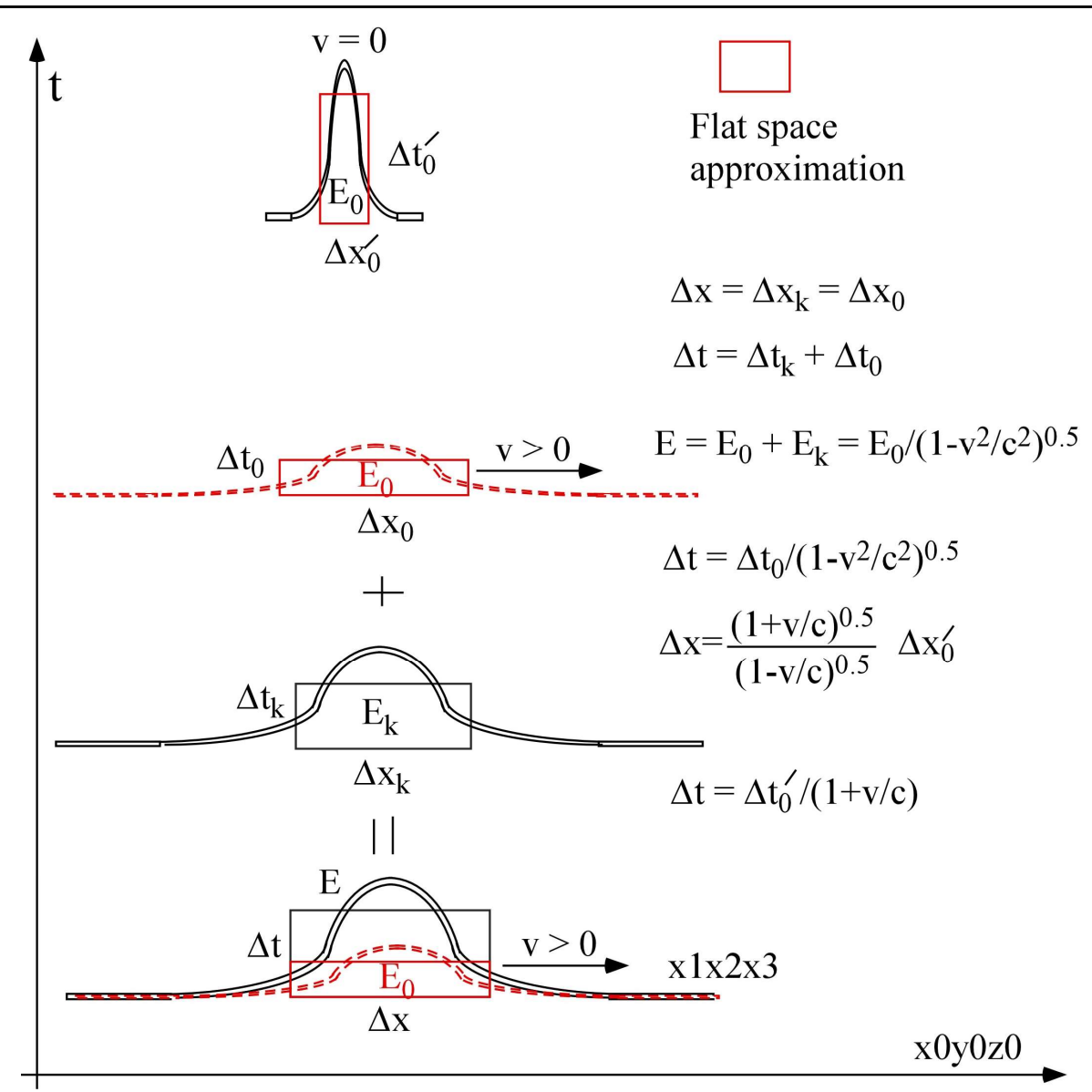

Fig. 7. Relativistic effect on the size of the particle with the non-zero rest mass. Also, see Fig. 4.

space and time space of the electron b-boson can be approximated by the flat space. The space wave function can be deduced from the Schrodinger equation of $\left(E-E_{0}\right) \psi(x)=H \psi(x)=$ $\left(\frac{p_{x}^{2}}{2 m}+V\right) \psi(x)$. The positive kinetic energy stands for the free electron with the plane space and time wave functions. Along with the constant potential energy of $\mathrm{V}<\mathrm{E}-\mathrm{E}_{0}$, the space and time wave functions of $\psi(x)=\frac{1}{\sqrt{\Delta x_{c}}} e^{\frac{i}{\hbar} \sqrt{2 m\left(\left(E-E_{0}-V\right)\right)} x}=\frac{1}{\sqrt{\Delta x_{c}}} e^{\frac{i}{\hbar} P_{x} x}$ and $\psi(t)=\frac{1}{\sqrt{c \Delta t_{c}}} e^{\frac{i E}{\hbar c} c t}$ can be used for an electron moving to the $+\mathrm{x}$ direction passing the potential wall. Here, $\mathrm{E}=\mathrm{c} \Delta \mathrm{t}_{\mathrm{c}} \Delta \mathrm{x}_{\mathrm{c}}$. The space wave function of $\psi(x)=\frac{1}{\sqrt{\Delta x_{c}}} e^{\frac{i}{\hbar} P_{x} x}$ is the solution of $E \psi(x)=H \psi(x)=\left(E_{0}+\frac{p_{x}^{2}}{2 m}+V\right) \psi(x)$ and $\left(E-E_{0}\right) \psi(x)=H \psi(x)=\left(\frac{p_{x}^{2}}{2 m}+V\right) \psi(x)$. The general solution considering the $+\mathrm{x}$ and $-\mathrm{x}$ moving directions of the free electron in this region is $\psi(x)=\frac{K}{\sqrt{\Delta x_{b c}}} e^{\frac{i}{\hbar} P_{x} x}+\frac{L}{\sqrt{\Delta x_{c r}}} e^{-\frac{i}{\hbar} P_{x} x}$ in Fig. 6. $\mathrm{K}$ and $\mathrm{L}$ are the coupling constants and $E_{S}=\left(E-E_{0}\right)=\left(\frac{p_{x}^{2}}{2 m}+V\right)$.

The free electron after going through the potential wall has the plane space and time wave functions of $\psi(x)=\frac{1}{\sqrt{\Delta x_{d}}} e^{\frac{i}{\hbar} P_{x} x}$ and $\psi(t)=\frac{1}{\sqrt{c \Delta t_{d}}} e^{\frac{i E}{\hbar c} c t}$ from the flat space approximation of the red box in 
the region D of Fig. 6. The coupling constant A, F, G, K and L can be calculated from the boundary conditions. When an electron moves to the $+\mathrm{x}$ direction on the $\mathrm{x} 1 \times 2 \times 3$ flat space out of the potential wall in the regions $\mathrm{B}$ and $\mathrm{D}$ of Fig. 6 , the time width is decreasing from $\Delta \mathrm{t}_{\mathrm{b}}$ to $\Delta \mathrm{t}_{\mathrm{d}}$ and the space width is increasing from $\Delta \mathrm{x}_{\mathrm{b}}$ to $\Delta \mathrm{x}_{\mathrm{d}}$. In Fig. $3, \mathrm{P}(\mathrm{x})=|\psi(\mathrm{x})|^{2}$ is the probability density at $\mathrm{x}$.

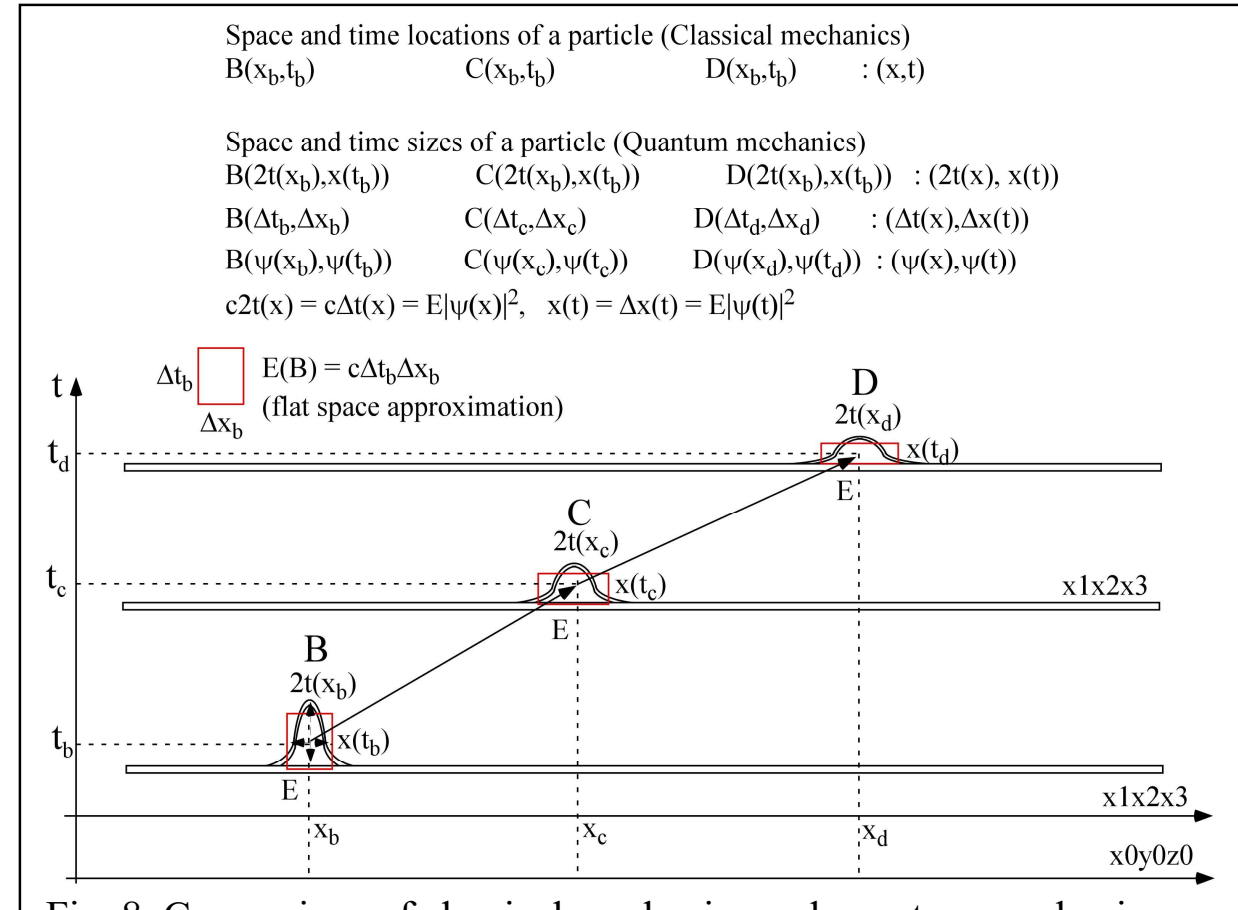

Fig. 8. Comparison of classical mechanics and quantum mechanics.

The electron tunneling through the potential wall barrier is explained by using the space expansion of the electron b-boson as shown in Fig. 6. Within the wall the electron cannot exist in the viewpoint of the classical mechanics. But in the viewpoint of the quantum mechanics the electron can exist within the potential wall with the reduced probability density at $\mathrm{x}$ obtained from the space expansion of the electron b-boson as shown in Figs. 6. After tunneling the potential wall, the electron wave function keeps the expanded space width and reduced time width which means the reduced probability density at $\mathrm{x}$ in Figs. 3, 4 and 6. Therefore, it should be noted that the b-boson shape is expanded along the space axis because of the energy increase by the relativistic effect when the electron is moving with a non-zero velocity.

In Fig. 4, the relation of the relativistic energy and wave functions in quantum mechanics for the $+q>0$ warped $x 1 \times 2 \times 3$ space is shown for the purpose of the simple summary of the quantum mechanics. The same explanation is applied for the $-\mathrm{q}<0$ warped $\mathrm{x} 1 \mathrm{x} 2 \times 3$ spaces by just vertically flipping the $\mathrm{x} 1 \mathrm{x} 2 \mathrm{x} 3$ spaces. The present procedure can be easily applied to the different potential walls with the different potential energies in Fig. 6. Note that in Fig. 7 the $\Delta x_{0}^{\prime}$ value is increased and $\Delta t_{0}^{\prime}$ is decreased with the increasing of the velocity because $\mathrm{E}_{0}$ is conserved. I solved the Schrodinger equations on the $\mathrm{x} 1 \times 2 \times 3$ space. The same procedure can be applied for the $\times 4 \times 5 \times 6$ and $\times 7 \times 8 \times 9$ spaces and this explanation can be extended easily to the $x 1 \times 2 \times 3-x 4 \times 5 \times 6$ and $\times 1 \times 2 \times 3-$ $\times 4 \times 5 \times 6-x 7 \times 8 \times 9$ spaces. In the present work, I added the physical meaning of the space and time wave functions. Then, the space and time wave functions can be obtained by solving the Schrodinger equations. Also, it is shown in Fig. 3 that $|\psi(\mathrm{x})|^{2},|\psi(\mathrm{t})|^{2}$ and $|\psi(\mathrm{x}, \mathrm{t})|^{2}=|\psi(\mathrm{x})|^{2}|\psi(\mathrm{t})|^{2}$, can be considered as the probability densities. It can be extended easily to the three-dimensional 
Schrodinger equation with $\Delta \mathrm{E}=\operatorname{ct}(\mathrm{x} 1, \mathrm{x} 2, \mathrm{x} 3) \Delta \mathrm{V}_{3}(\mathrm{x} 1 \mathrm{x} 2 \mathrm{x} 3)=\mathrm{E}|\psi(\mathrm{x} 1, \mathrm{x} 2, \mathrm{x} 3)|^{2} \Delta \mathrm{V}_{3}(\mathrm{x} 1 \mathrm{x} 2 \mathrm{x} 3)$ in Fig. 3. $\mathrm{V}_{3}(\mathrm{x} 1 \mathrm{x} 2 \mathrm{x} 3)$ is the three-dimensional space volume. Schrodinger equation has been derived for the matter with the positive energies of $\mathrm{E}_{0}>0$ and $\mathrm{E}>0$ in the present work. In other words, it has been used for the positive space momenta and positive time momentum in the present work. Because the wave function comes from the description of the space-time shapes, the same Schrodinger equations and the derived wave functions can be generally used in order to describe all of flat and warped spaces including the flat spaces, matters (particles) and antimatters (antiparticles) with the positive energy or negative energy. For the partner spaces with the negative energy, the negative energies of $\mathrm{E}_{0}<0$ and $\mathrm{E}<0$ need to be used in the Schrodinger equations and the derived wave functions. Space momenta $\left(\mathrm{p}_{\mathrm{x}}\right)$ can have the positive and negative value. Note that the negative energy corresponds to the negative mass. For the high velocity case, the revised Schrodinger equation of $E \psi(x)=H \psi(x)=\left(E_{0}+\frac{p_{x}^{2}}{2 m}+\frac{3 p_{x}^{4}}{8 m_{0}^{3} c^{2}}+V(x)\right) \psi(x)$ needs to be used because it gives the better results than the Schrodinger equation of $E \psi(x)=H \psi(x)=$ $\left(E_{0}+\frac{p_{x}^{2}}{2 m}+V(x)\right) \psi(x)$ as shown in Fig. 5. It is shown in the present work that the Schrodinger equation can be obtained from the relativistic energy equation of $E=\mathrm{E}_{0} /\left(1-\mathrm{v}^{2} / \mathrm{c}^{2}\right)^{0.5}$.

\subsection{Classical mechanics, relativity theory and quantum mechanics}

As shown in Fig. 8, the space and time locations of a particle can be described as $(\mathrm{x}, \mathrm{t})$ in terms of the classical mechanics which the elementary particle is treated as the point particle. In Fig. 8, the space and time locations of a particle are described on the $\mathrm{x}$ coordinates of $(\mathrm{x} 1, \mathrm{x} 2, \mathrm{x} 3)$ for the simplicity in the present explanation. Generally, the coordinates of $\mathrm{x}$ can stand for the $((\mathrm{x} 0, \mathrm{y} 0$, $\mathrm{z} 0),(\mathrm{x} 1, \mathrm{x} 2, \mathrm{x} 3),(\mathrm{x} 4, \mathrm{x} 5, \mathrm{x} 6),(\mathrm{x} 7, \mathrm{x} 8, \mathrm{x} 9))$ in our three-dimensional quantized spaces. It has been discussed in the present work that the particle really has the space and time sizes [1]. Therefore, we need to know the particle sizes and locations in the space and time geometry. The space and time sizes of the particle are closely connected to the space and time wave functions in quantum mechanics as discussed in the present work. Therefore, the particle space and time sizes can be described as $(\psi(\mathrm{x}), \psi(\mathrm{x})),(\Delta \mathrm{t}(\mathrm{x}), \Delta \mathrm{x}(\mathrm{t}))$ or $(2 \mathrm{t}(\mathrm{x}), \mathrm{x}(\mathrm{t}))$. Therefore, the classical mechanics is about the change of the space and time locations of the particles and the quantum mechanics is about the change of the space and time sizes of the particles. The particle size is, really, the size of the particle b-boson as shown in Fig. 6. The particle matter with the Planck size is moving within the particle b-boson as shown in Fig. 6. 


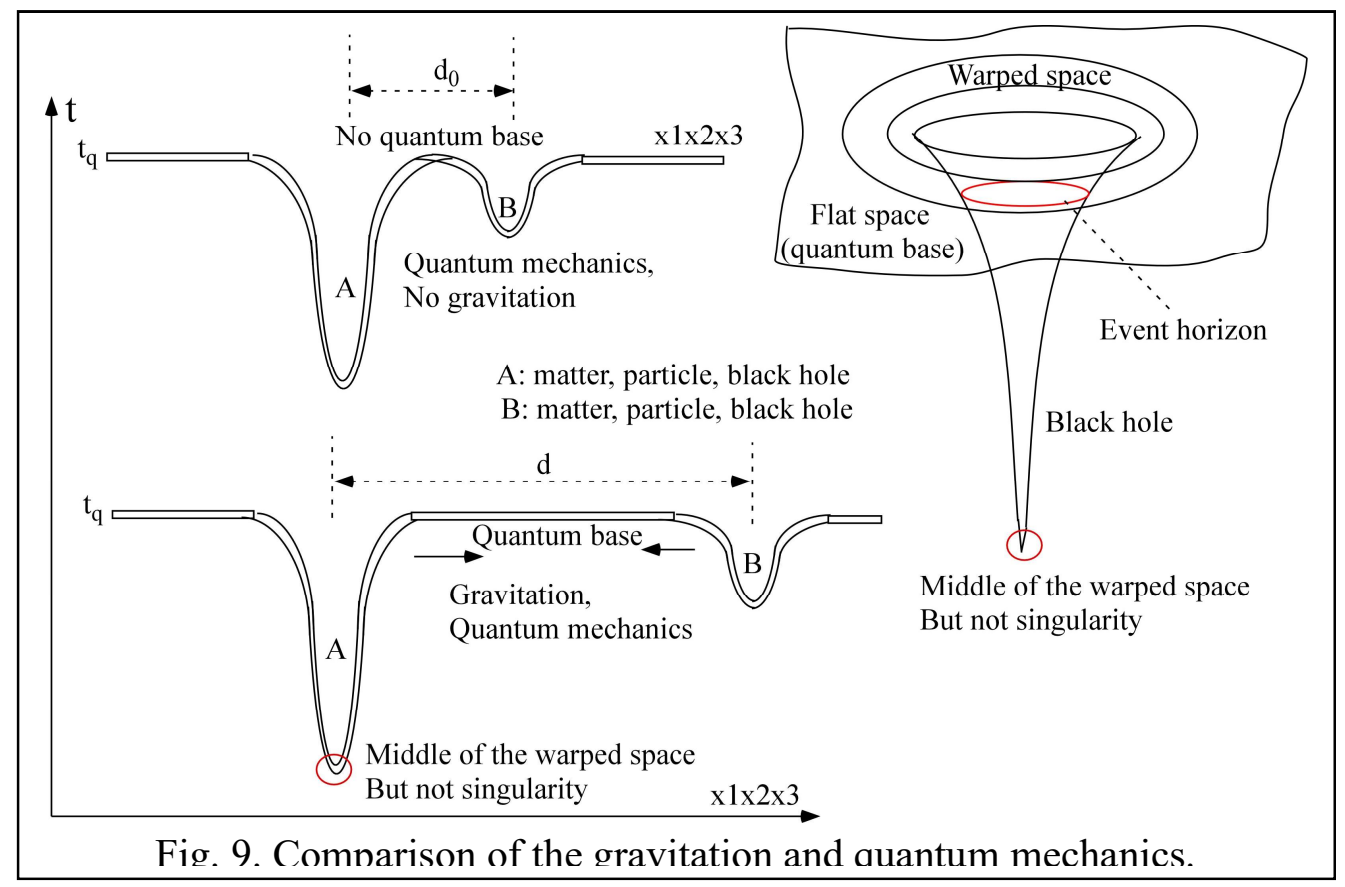

Generally, the particle b-bosons belong to the warped spaces. The warped spaces have the spacetime coordinates expressed as $(\mathrm{x}, \mathrm{t})$ and space-time sizes expressed as $((\psi(\mathrm{x}), \psi(\mathrm{x})),(\Delta \mathrm{t}(\mathrm{x}), \Delta \mathrm{x}(\mathrm{t}))$ or $(t(x), x(t))$. Generally, the coordinates of $x$ stand for the $((x 1, x 2, x 3),(x 4, x 5, x 6)$ and $(x 7, x 8, x 9))$ in our three-dimensional quantized spaces. These space-time coordinates are defined as the center space and time locations of the particles in Fig. 7. Therefore, there are two kinds of changes for the warped matters. One is the change of the space-time coordinates (momentum transition) and another one is the change of the space-time sizes (energy transition). The study on the change of the space-time coordinates of the warped spaces can be called as the classical mechanics. The study on the change of the space-time sizes of the warped spaces can be called as the quantum mechanics. Because we have applied the quantum mechanics only to the elementary particles with the diameter sizes less than $\sim 10^{-15} \mathrm{~m}$, it has been misunderstood that the quantum mechanics works only for the small scales. However, generally the quantum mechanics should work for all sizes of the warped spaces including all the matters and particles. Also, the space-time sizes of the flat spaces have been described by the plane waves which are closely connected to the quantum mechanics in the present work. The space-time coordinates of $(\mathrm{x}, \mathrm{t})$ which are closely connected to the classical mechanics can be properly assigned to these flat spaces, too. Then, the quantum mechanics and classical mechanics can be applied to the flat spaces, too. It means that our whole universes should be described by both of the quantum mechanics and the classical mechanics.

The b-boson and the internal matter of a particle in Fig. 6 can be separated near the black hole. If the b-boson and internal matter of a particle is separated, the b-boson and the internal matter are changed to the flat space or absorbed (merged) into the warped space of the black hole. Then the quantum mechanics and classical mechanics cannot be applied to the particle because the spacetime location of $(\mathrm{x}, \mathrm{t})$ and the space-time size of $((\psi(\mathrm{x}), \psi(\mathrm{x})),(\Delta \mathrm{t}(\mathrm{x}), \Delta \mathrm{x}(\mathrm{t}))$ or $(\mathrm{t}(\mathrm{x}), \mathrm{x}(\mathrm{t}))$ cannot be defined any more to the collapsed particle which loses its identity. The boundary outline of the black hole is the event horizon of the present work as explained at the next two paragraphs of this section. The quantum mechanics and classical mechanics should be applied to the new merged 
state of the black hole. Therefore, the gravitational formula can be applied only to the space length larger than the space size of the particle as shown in Fig. 9. It will remove the singularity in the gravitational interaction of the black hole. Because the quantum mechanics is based on the spacetime sizes of the particle in Fig. 8, the space and time locations of the particle guessed in terms of the quantum mechanics have always the uncertainties of $\Delta x$ and $\Delta t$, respectively. But the space and time locations of the particle in the classical mechanics are expressed as the average center values of $\mathrm{x}$ and $\mathrm{t}$, respectively, in Fig. 8 because the classical mechanics is based on the space and time locations of the point-like particle. It implies that the classical mechanics is based on the concept of the point particle and the quantum mechanics is based on the non-zero size concept (non-zero space and time sizes) of the particle.

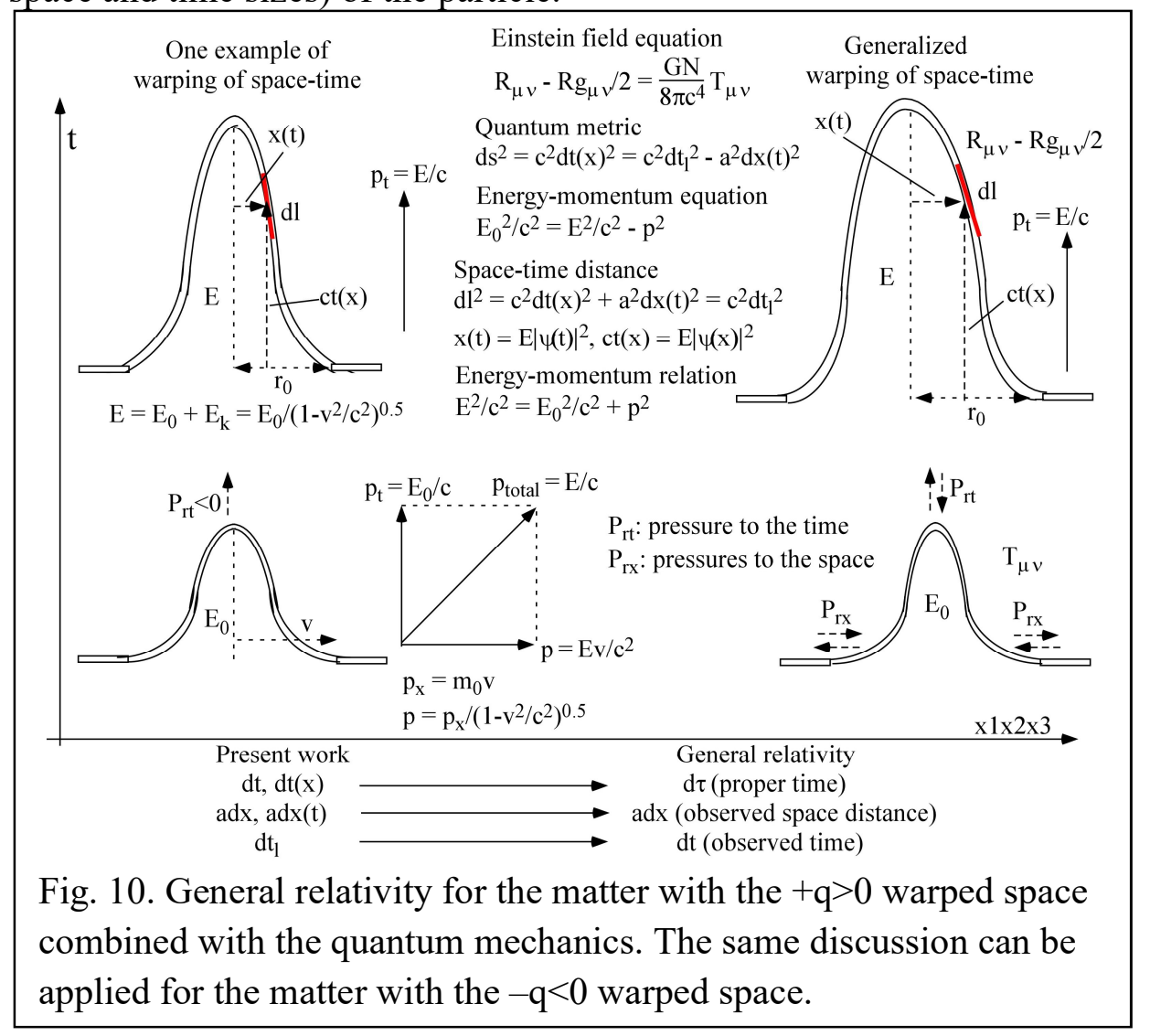

In Fig. 9, two matters of $\mathrm{A}$ and $\mathrm{B}$ with the distance of $\mathrm{d}$ are approaching to each other by the gravitational force. These two matters including particles and black holes have the warped $\times 1 \times 2 \times 3$ spaces. These two matters can be the $\mathrm{x} 1 \times 2 \times 3$, $\mathrm{x} 1 \times 2 \times 3-\mathrm{x} 4 \times 5 \times 6$ or $\times 1 \times 2 \times 3-\times 4 \times 5 \times 6-\times 7 \times 8 \times 9$ matters including particles and black holes which have the warped $\times 1 \times 2 \times 3$ spaces. The matters are moving on the quantum bases which means the $\times 1 \times 2 \times 3$ flat space and the gravitons are moving on the quantum bases, called as the graviton bases, between these two matters of A and B. In this case, the quantum mechanics and gravitation can be used to describe the dynamics of two matters. But when the warped spaces of two mattes are touching at the distance $\mathrm{d}_{0}$, the space between two matters is not the flat space but the warped space. Then the gravitation cannot be applied at the distance less than $\mathrm{d}_{0}$ because there is no graviton base. It blocks the gravitational collapse which leads to the gravitational singularity at $d=0$. The combined (merged) warped space shape of two matters with the distance of $\mathrm{d}<\mathrm{d}_{0}$ can be described by the quantum mechanics but not by the gravitation. Two matters lose their independent identities by changing to the combined (merged) 
warped space shape of two matters. The combined (merged) warped space shape of two matters can be described by combining two wave functions of two matters by using the quantum mechanics. Therefore, it is shown in the present work that the gravitational singularity cannot exist as shown in Fig. 9.

In the present work, the event horizon of the black hole is redefined as the space boundary within which the warped space of the incoming matter to the black hole loses its identity completely by being absorbed into the warped space of the black hole. In the warped region out of the event horizon of the black hole, the warped space of the incoming matter to the black hole loses its identity partly by being absorbed into the warped space of the black hole. Many kinds of particles and antiparticles are created by the quantum warped space fluctuations around the outside of the

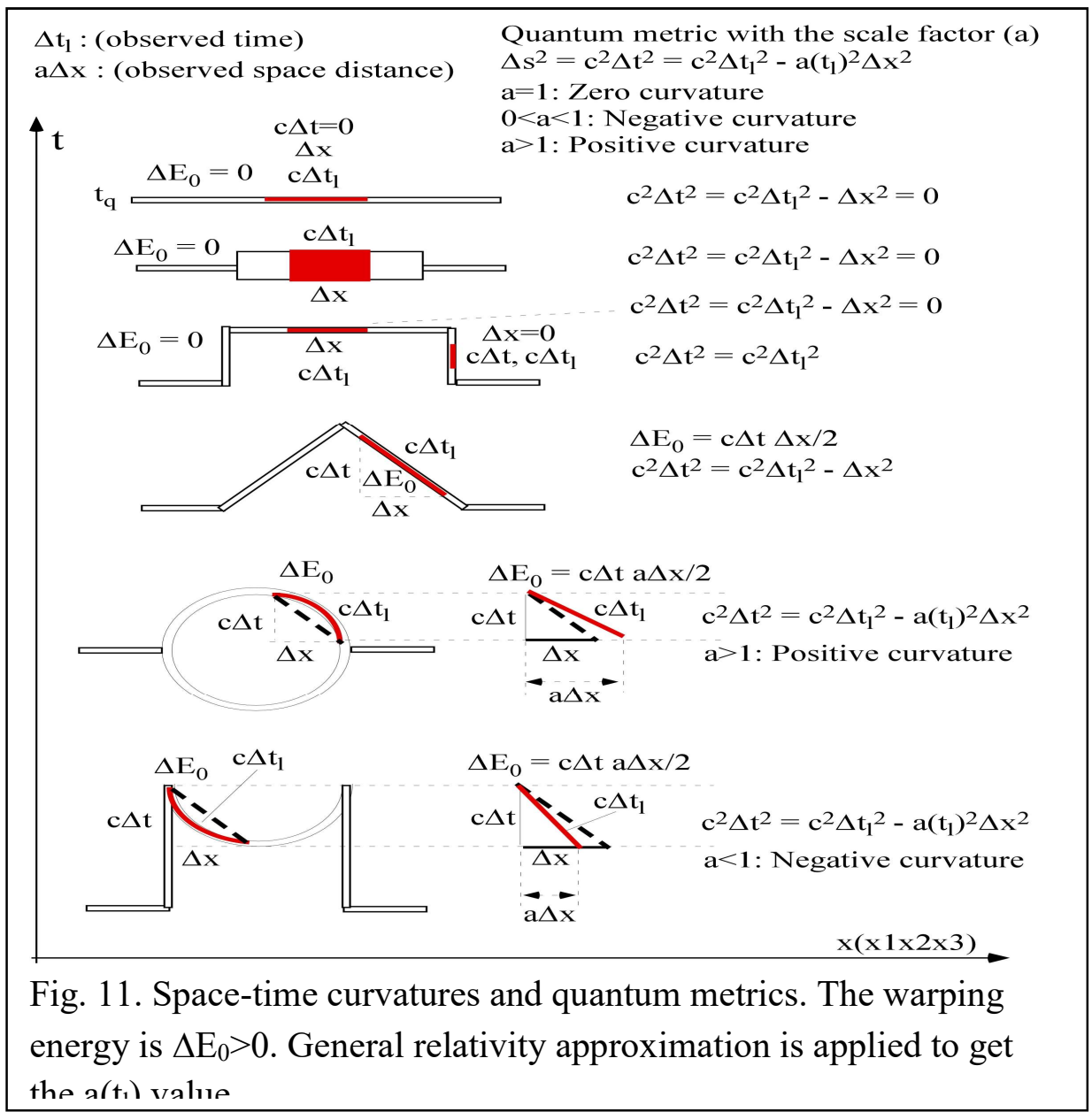

event horizon of the black hole. When the warped space part of the incoming matter stays in the warped region out of the event horizon, other matters including the information of the incoming matter can be created and emitted. The $\mathrm{x} 1 \mathrm{x} 2 \times 3$, $\mathrm{x} 1 \times 2 \times 3-\mathrm{x} 4 \times 5 \times 6$ or $\mathrm{x} 1 \times 2 \times 3-\mathrm{x} 4 \times 5 \times 6-\mathrm{x} 7 \mathrm{x} 8 \mathrm{x} 9$ matters (particles, black holes) have the warped $\mathrm{x} 1 \times 2 \times 3$ spaces [1]. In other words, the $\times 1 \times 2 \times 3$ spaces associated with the $\mathrm{x} 1 \times 2 \times 3, \mathrm{x} 1 \times 2 \times 3-\mathrm{x} 4 \times 5 \times 6$ or $\mathrm{x} 1 \times 2 \times 3-\mathrm{x} 4 \times 5 \times 6-\mathrm{x} 7 \mathrm{x} 8 \times 9$ matters (particles, black holes) are warped. Then, other $\times 4 \times 5 \times 6$ and $\times 7 \times 8 \times 9$ spaces associated with the $x 1 \times 2 \times 3$ space are warped at the same way as the $\mathrm{x} 1 \times 2 \times 3$ space. Because the photons are moving on the quantum bases (called as the photon bases) with the flat space, the paths of the photons are curved on the space by going around the matters in order to follow the quantum bases but not the warped spaces. 
This is called the lensing effect of the matters on the photons. Photons cannot exist within the event horizon of the black hole because there is no quantum base with the flat space within the black hole. Also, because the gravitation does not exist within the black hole, there is no gravitational singularity in terms of the present three-dimensional quantized space model. The black hole is just the hugely warped space with the event horizon defined in the present work. The $x 1 \times 2 \times 3$ matter can be described by the warped $\mathrm{x} 1 \times 2 \times 3$ space. The more energy the $\mathrm{x} 1 \times 2 \times 3$ matter has, the more warped the $x 1 \times 2 \times 3$ space associated with the $x 1 \times 2 \times 3$ matter is. The more energy the $x 1 \times 2 \times 3$ matter has, the more gravitational effect the $\mathrm{x} 1 \times 2 \times 3$ matter associated with the warped $\times 1 \times 2 \times 3$ space has. The particle or the matter moves on the flat $x 1 \times 2 \times 3$ space but not within the warped $\mathrm{x} 1 \times 2 \times 3$ space. Because the particle or the matter is the warped $\mathrm{x} 1 \times 2 \times 3$ space, the particle or the matter cannot move on the warped $\times 1 \times 2 \times 3$ space by keeping its identity. The gravitation makes

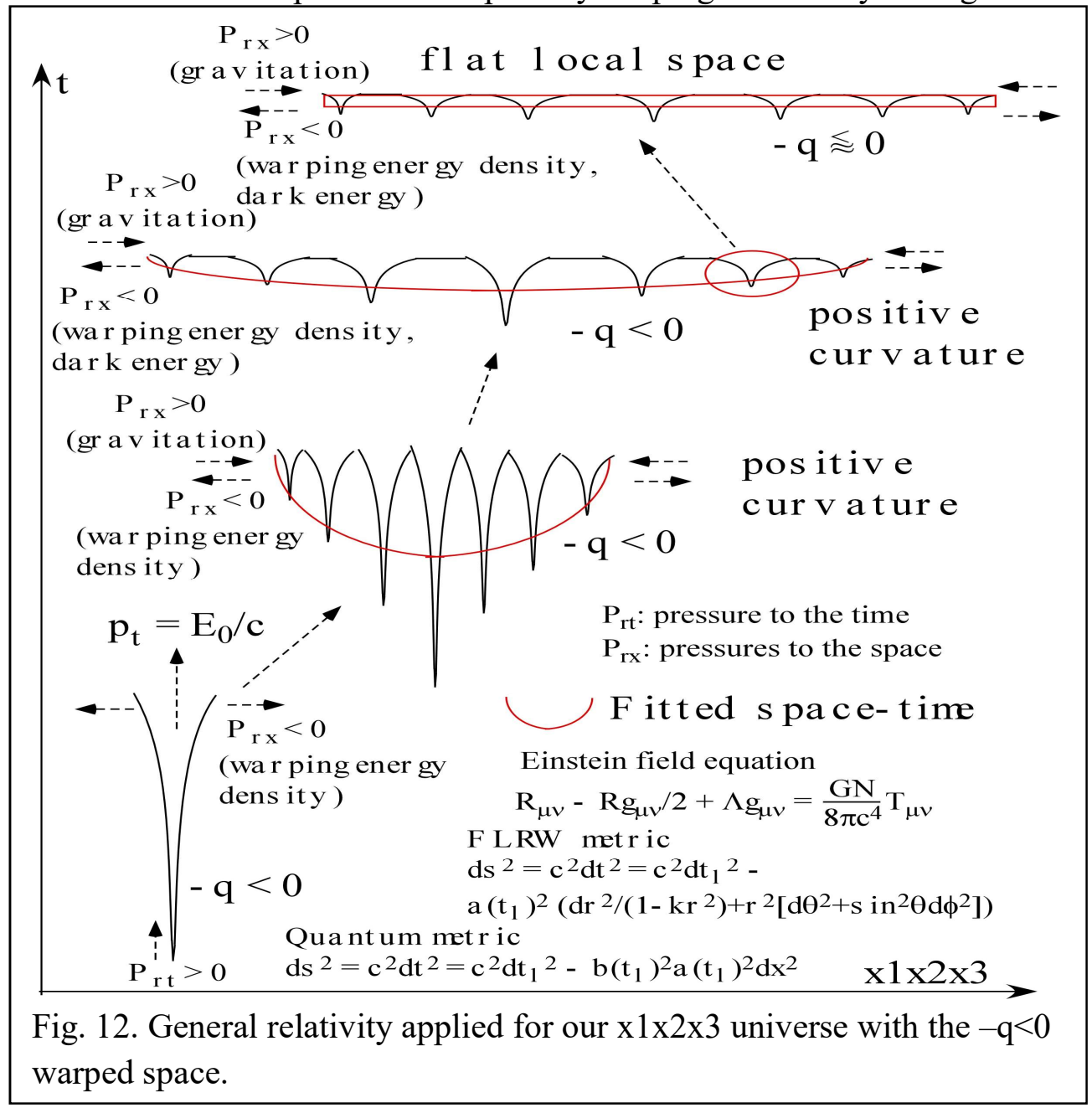

sense only when the particle or the matter moves on the flat $\mathrm{x} 1 \times 2 \times 3$ space.

First, note that the time definition in the present work is different from the time definition of the special and general relativity theories as shown in Fig. 10. The proper time $(\tau)$ and observed time $(t)$ in the special and general relativity theories correspond to the proper time $(t)$ and observable time $\left(\mathrm{t}_{1}\right)$, respectively, in the present work. In the general relativity, the curvature of the matter is related to the energy, space momenta and possible other stresses by using the following Einstein field equation. $\mathrm{R}_{\mu v}-\mathrm{Rg}_{\mu v} / 2=\mathrm{GNT}_{\mu v} /\left(8 \mathrm{pc}^{4}\right)$. The left terms of this equation expresses the curvature 
of the space-time shape of the matter and the right term has the energy-momentum tensor (energymementum-stress tensor) of $\mathrm{T}_{\mu \nu}$. The Schwarzschard solution (metric) considering the gravitation effect on the curvature of the space cannot be used for the region with $r<d_{0}$ in Fig. 9. This condition removes the possibility of the black hole with the singularity. The space-time curvature of the matter can be described by using the quantum metric given in the present work because the space-time shape or curvature of the matter should be described by using the Schrodinger equation of quantum mechanics which gives the quantum metric of $\mathrm{ds}^{2}=\mathrm{c}^{2} \mathrm{dt}(\mathrm{x})^{2}=\mathrm{c}^{2} \mathrm{dt}_{1}(\mathrm{x})^{2}-\mathrm{dx}(\mathrm{t})^{2}$ in Figs 10 and 11. In quantum mechanics, $\operatorname{ct}(\mathrm{x})=\mathrm{E}|\psi(\mathrm{x})|^{2}$ and $\mathrm{x}(\mathrm{t})=\mathrm{E}|\psi(\mathrm{t})|^{2}$ are the space energy density $\left(\rho_{x}\right)$ and time energy density $\left(\rho_{t}\right)$, respectively in Fig. 3. Three examples of possible shapes of the $\mathrm{x} 1 \times 2 \times 3$ matter having the zero curvature, positive curvature and negative curvature are shown in Fig. 11. For example, the b-boson has the spherical shape with the positive curvature. The flat space has the zero curvature. The quantum metric with the curvature scale factor $\left(\mathrm{a}\left(\mathrm{t}_{1}\right)\right)$ is $\Delta \mathrm{s}^{2}=$

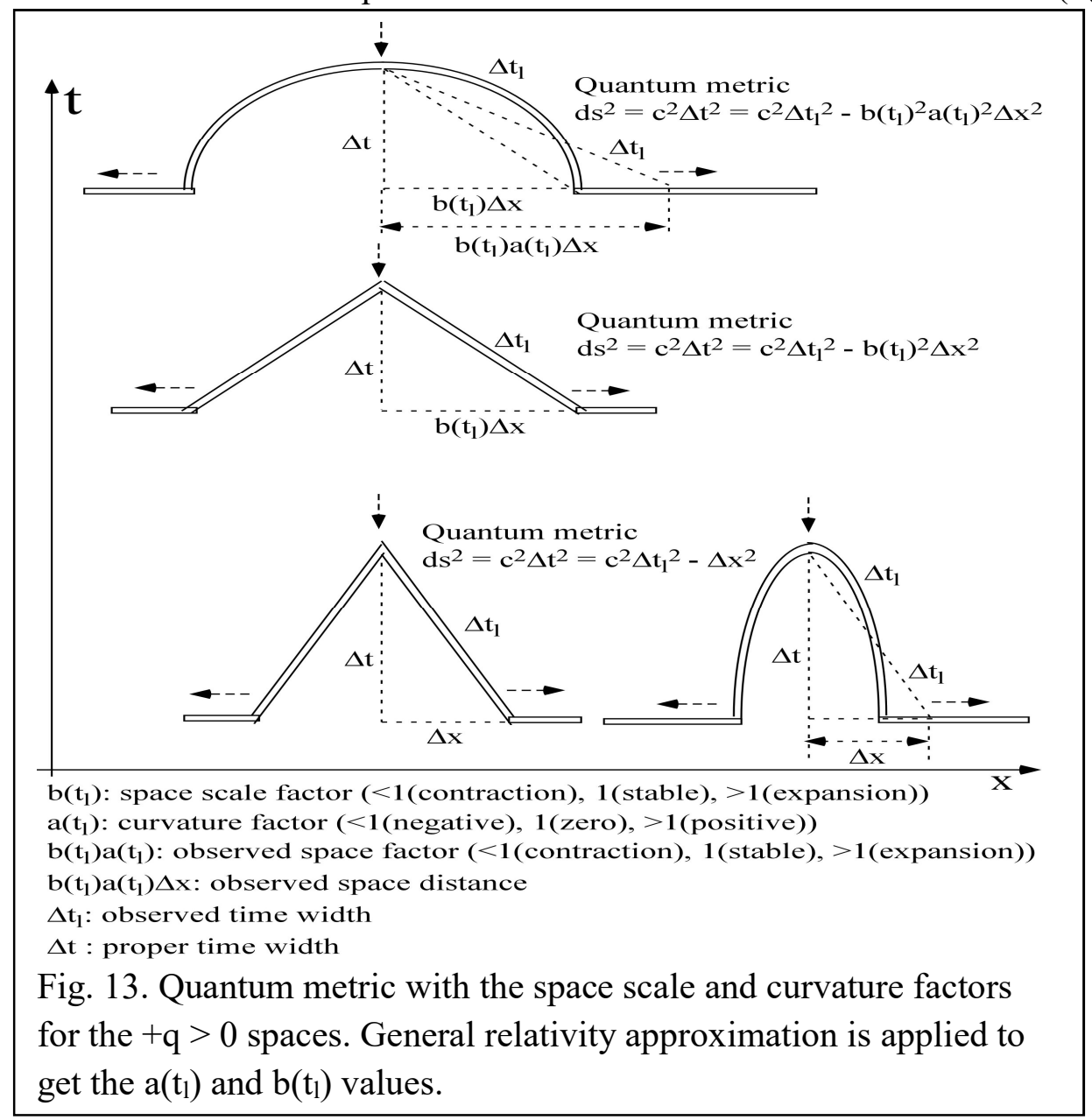

$\mathrm{c}^{2} \Delta \mathrm{t}^{2}=\mathrm{c}^{2} \Delta \mathrm{t}^{2}-\mathrm{a}\left(\mathrm{t}_{1}\right)^{2} \Delta \mathrm{x}^{2}$. The values of $\mathrm{a}=1,0<\mathrm{a}<1$ and $\mathrm{a}>1$ correspond to the zero, negative and positive curvatures, respectively.

For the $\mathrm{x} 1 \mathrm{x} 2 \mathrm{x} 3$ universe shape, the Einstein field equation and quantum mechanics can be used together as shown in Fig. 12. In order to describe the space-time shape of the universe, the contributions from the $\times 4 \times 5 \times 6$ and $\times 7 \times 8 \times 9$ matters are disregarded for the simplicity because the $\mathrm{x} 1 \times 2 \times 3$ matters associated to the $\mathrm{x} 4 \times 5 \times 6$ and $\times 7 \times 8 \times 9$ matters are relatively small compared to the $\mathrm{x} 1 \times 2 \times 3$ matters. The matters with the negative electric charge of $-\mathrm{q}<0$ are dominating in our 
$\mathrm{x} 1 \times 2 \times 3$ matter universe. The $\mathrm{x} 1 \times 2 \times 3$ matter universe with the positive energy was created as the $\mathrm{x} 1 \times 2 \times 3$ matter of the huge black hole in Fig. 12. It is called as the big bang. This black hole has been rapidly expanded to the space direction and rapidly contracted to the time direction toward to the flat space because the flat space is the most stable shape of the space-time with the minimum energy density. In other words, the warping energy density of $\rho(x)=E|\psi(x)|^{2}=\operatorname{ct}(x)(\sim \Delta t)$ is rapidly decreased in a very short time and the space width $(\sim \Delta \mathrm{x})$ is rapidly increased in a very short time because $\mathrm{E}=\mathrm{c} \Delta \mathrm{t} \Delta \mathrm{x}$ in the flat space approximation is conserved. It is thought that this warping energy effect causes the inflation of the universe. It has created many small $\times 1 \times 2 \times 3$ matters. Currently, the universe is made of $x 1 \times 2 \times 3$ matters, $x 1 \times 2 \times 3-x 4 \times 5 \times 6$ and $\times 1 \times 2 \times 3-x 4 \times 5 \times 6-$ $\mathrm{x} 7 \mathrm{x} 8 \mathrm{x} 9$ matters. The $\mathrm{x} 1 \times 2 \times 3$ matters are dominating in the universe scale. Even in the local scale of the galaxy cluster, it is proposed in the present work that the $\mathrm{x} 1 \mathrm{x} 2 \mathrm{x} 3$ matters like the B1 dark matters and $\times 1 \times 2 \times 3$ black holes are dominating over the $\times 1 \times 2 \times 3-x 4 \times 5 \times 6$ and $\times 1 \times 2 \times 3-x 4 \times 5 \times 6-$ $\mathrm{x} 7 \mathrm{x} 8 \mathrm{x} 9$ matters like the leptons, hadrons [1]. All matters are moving in the flat $\mathrm{x} 1 \mathrm{x} 2 \mathrm{x} 3 \mathrm{space}$. But the fitted space-time has the curvature as shown in Fig. 12. This fitted warped space of the whole x 1x2x3 universe has the negative electric charge warping. However the present local space can

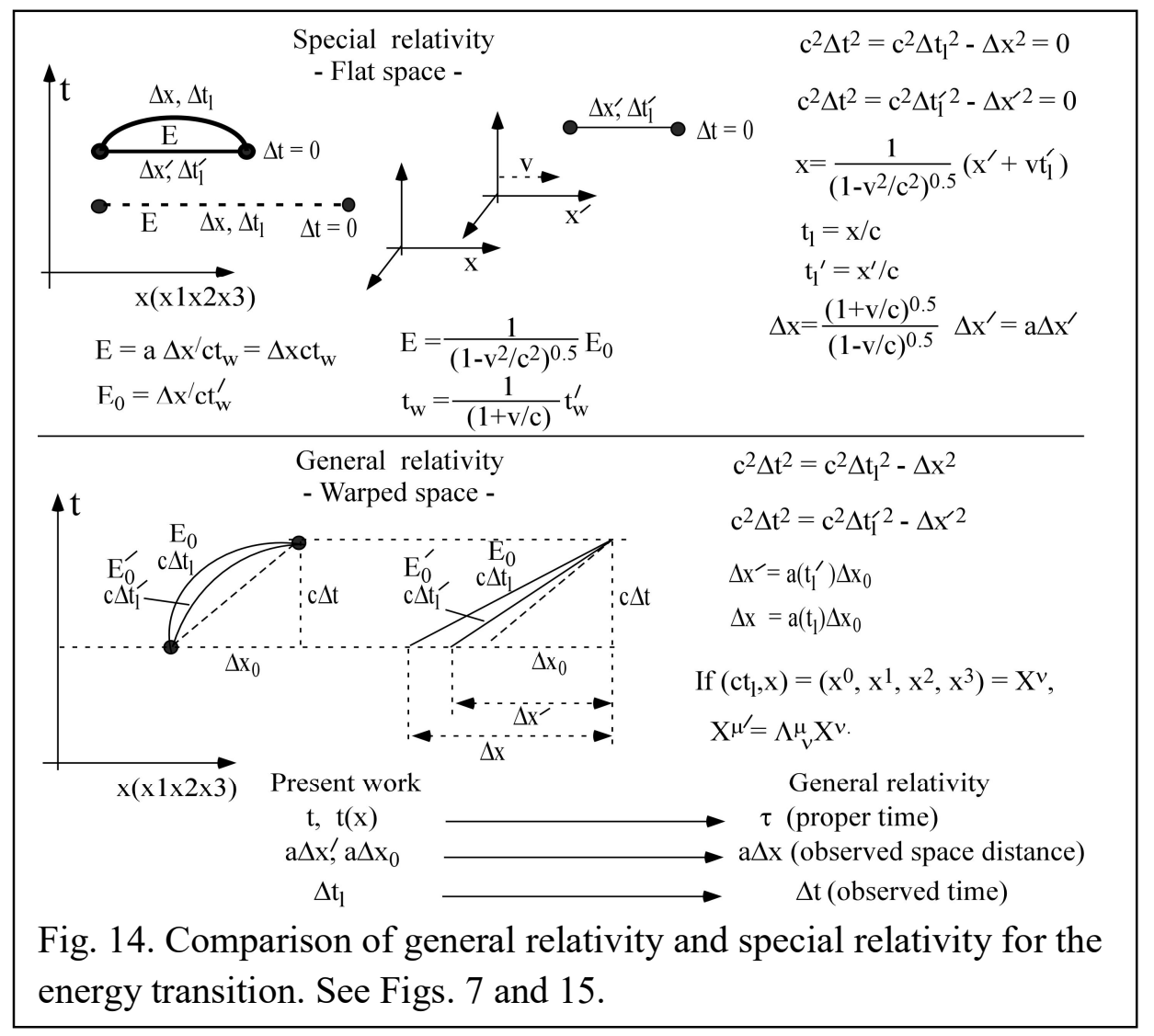

have the nearly flat fitted space. The curvature of this fitted space depends on the gravitations of the matters including the dark matters and normal matters to give the positive space pressure and the space expansion caused by the newly added $\mathrm{x} 1 \times 2 \times 3$ spaces to give the negative space pressure (called as the dark energy). Therefore, the additional dark and warping energy term of $\Lambda g_{\mu \nu}$ can be included into the Einstein field equation in Fig. 12. 
Also, it needs to be noted that the warping energy density which plays a major role during the inflation time of the universe gives the negative space pressure. Because the present local space is believed to be nearly flat, it is concluded that the dark energy on the accelerated space expansion of the present local universe is increasing more and more. Those effects can be separated into the space factor of $b(t)$ due to the dark energy and curvature factor of $a(t)$ due to the warping energy

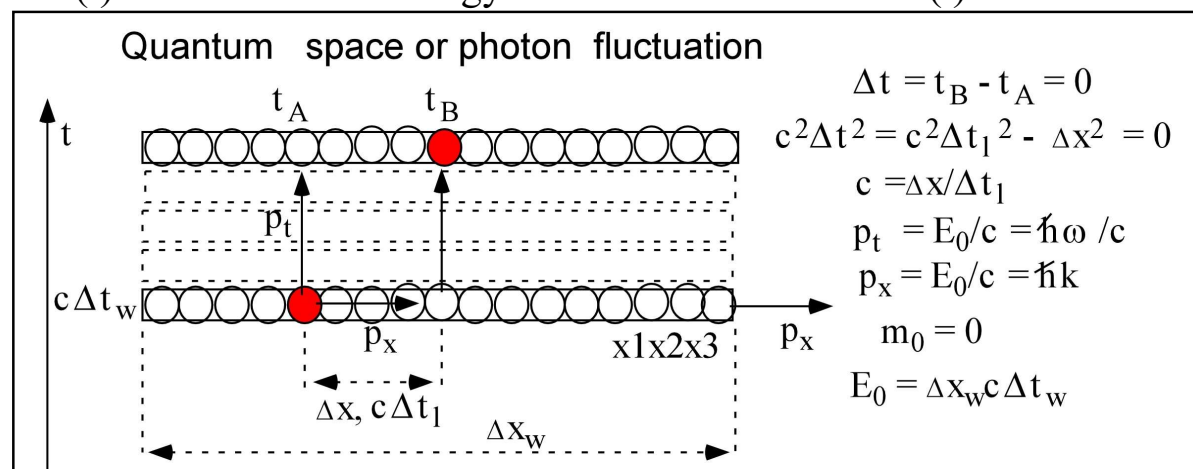

The information of the energy and space momenta is transferred by the quantum space or photon fluctuation.

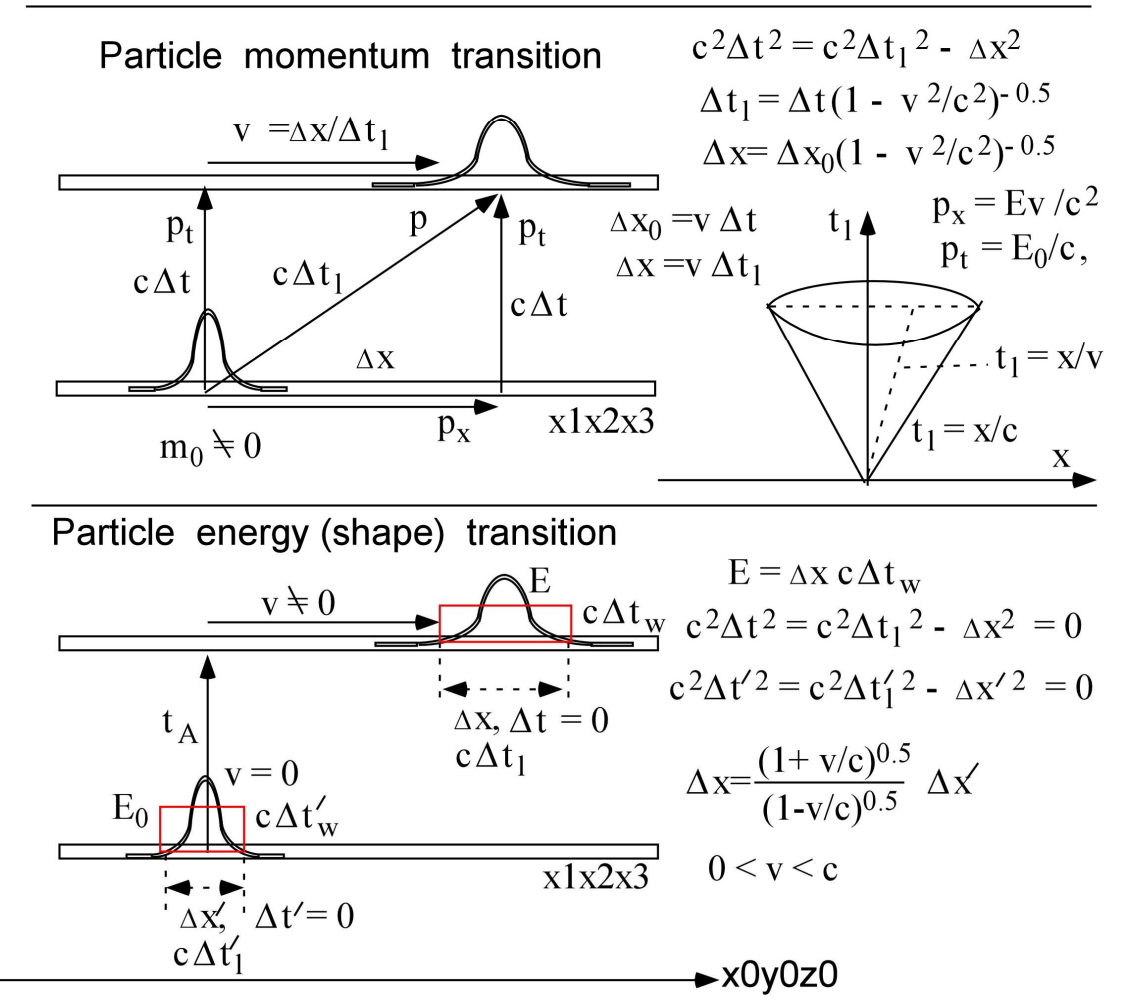

Fig. 15. The energy and space-time momentum transitions of the photon. auantum space and particle are compared.

in the quantum metrics in Figs. 12 and 13. The general solution of the Einstein field equation with the dark energy (+ warping energy) term of $\Lambda \mathrm{g}_{\mu \nu}$ is the FLRW metric (Friedmann-LemaitreRobertson-Walker metric) as shown in Fig. 12. This FLRW metric corresponds to the quantum metric of $\mathrm{ds}^{2}=\mathrm{c}^{2} \mathrm{dt}^{2}=\mathrm{c}^{2} \mathrm{dt}_{1}^{2}-\mathrm{b}\left(\mathrm{t}_{1}\right)^{2} \mathrm{a}\left(\mathrm{t}_{1}\right)^{2} \mathrm{dx}^{2}$. The space scale factor $\left(b\left(t_{1}\right)\right)$ responsible for the space expansion and curvature scale factor $\left(\mathrm{a}\left(\mathrm{t}_{1}\right)\right)$ responsible for the additional curvature are included in the quantum metric as shown in Fig. 12. Here the total energy $\left(E_{0}\right)$ of the whole universe is conserved through the evolution of the whole universe. Our universe has the positive curvature as 
shown in Fig. 12 and the negative charge. The FLRW metric can be used in order to describe the local fitted universe. The factor of $a\left(t_{1}\right)$ is called as the (space) scale factor and the curvature of the local fitted universe can be related to the k value within the FLRW metric. Generally the given values of $\mathrm{k}=-1,0$ and 1 represent the local fitted universe which have the negative, zero and positive curvatures, respectively. Our local fitted universe has the $k=1$ value because it has the positive curvature and $-\mathrm{q}<0$ warping as shown in Fig. 13.

In Fig. 13, the quantum metric is related with the space expansion and space curvature. Our universe has the space expansion. And it has the positive curvature with the negative charge. In order to describe the geometry of our universe in Fig. 12, the quantum metric with the space scale factor $\left(b\left(t_{1}\right)\right)$ and curvature factor $\left(a\left(t_{1}\right)\right)$ is needed as shown in Fig. 13. The space with the positive curvature and the $+\mathrm{q}>0$ warping are shown in Fig. 13 for the explanation. The same discussion can be applied to the space with the positive curvature and the $-\mathrm{q}<0$ warping in Fig. 12. The quantum metric can be compared with the FLRW metric (Friedmann-Lemaitre-Robertson-Walker metric) as shown in Fig. 12. The space scale factor $\left(b\left(t_{1}\right)\right)$ in Fig. 13 is expressed as a $\left(t_{1}\right)$ in FLRW metric. And the curvature factor $\left(\mathrm{a}\left(\mathrm{t}_{1}\right)\right)$ in Figs. 11 and 13 is expressed as the terms including the factor of $1 /\left(1-\mathrm{kr}^{2}\right)$ in FLRW metric. In FLRW metric, the spaces with the negative, zero and positive curvatures are defined as the space with the $\mathrm{k}=-1,0$ and +1 values, respectively. Note that the local space in our universe is flat as shown in Fig. 11. Therefore, $a(t l)=1$ in the quantum metric of Fig. 13 and $\mathrm{k}=0$ in FLRW metric can be roughly used for the local flat space.

The comparison of the special and general relativities are compared in Fig. 14. First the special relativity is applied to the flat space. The $\mathrm{x}^{\prime}$ space is moving with the velocity of $\mathrm{v}$ relative to the $\mathrm{x}$ space. Then, the flat space with the space distance of $\Delta \mathrm{x}^{\prime}$ has the observed time of $\Delta \mathrm{t}_{1}^{\prime}=\Delta \mathrm{x}^{\prime} / \mathrm{c}$ in Fig. 14. Note that the proper time $(\tau)$ and observed time $(\mathrm{t})$ in the special and general relativity theories correspond to the proper time $(t)$ and observable time $\left(t_{1}\right)$ in the present work. In the flat space, $\Delta t=0$. The space seen from the $\mathrm{x}$ space is warped because of the velocity effect of $\mathrm{v}$ as shown in Fig. 14. Then, the space distance $(\Delta \mathrm{x})$ and time $\left(\Delta \mathrm{t}_{\mathrm{l}}\right)$ observed from the $\mathrm{x}$ space by following the warped space-time path are changed from the space distance $\left(\Delta \mathrm{x}^{\prime}\right)$ and time $\left(\Delta \mathrm{t}_{1}{ }^{\prime}\right)$ observed from the $\mathrm{x}^{\prime}$ space by following the flat space-time. Then, the warped space with the space distance of $\Delta \mathrm{x}$ has the observed time of $\Delta \mathrm{t}_{1}=\Delta \mathrm{x} / \mathrm{c}$ in Fig. 14. Therefore, the quantum metrics of $\Delta \mathrm{s}^{2}=\mathrm{c}^{2} \Delta \mathrm{t}^{2}=\mathrm{c}^{2} \Delta \mathrm{t}_{1}^{2}-\Delta \mathrm{x}^{2}=0$ and $\Delta \mathrm{s}^{2}=\mathrm{c}^{2} \Delta \mathrm{t}^{2}=\mathrm{c}^{2} \Delta \mathrm{t}_{1}{ }^{2}-\Delta \mathrm{x}^{/ 2}$ can be used. The Lorentz transformation between the $\mathrm{x}$ and $\mathrm{x}^{\prime}$ space can be derived as follows. In special relativity, $\mathrm{x}=\gamma \mathrm{x}^{\prime}$ $+\mathrm{bt}_{1}^{\prime}$ and $\gamma>0$. When $\mathrm{x}=0, \mathrm{x}^{\prime}=-\mathrm{vt}_{1}{ }^{\prime}$. Then, $-\gamma \mathrm{vt_{1 }}{ }^{\prime}+\mathrm{bt}_{1}{ }^{\prime}=0$ and $\mathrm{b}=\gamma \mathrm{v}$. And $\mathrm{x}=\gamma\left(\mathrm{x}^{\prime}+\mathrm{vt}_{1}{ }^{\prime}\right)$. By the same way, $\mathrm{x}^{\prime}=\gamma\left(\mathrm{x}-\mathrm{vt}_{1}\right)$. As shown in the quantum metrics of Fig. 12, $\mathrm{x}=\mathrm{ct}_{1}$ and $\mathrm{x}^{\prime}=\mathrm{ct}_{1}{ }^{\prime}$. And $\mathrm{ct}_{1}$ $=\mathrm{c} \gamma(1+\mathrm{v} / \mathrm{c}) \mathrm{t}_{1}{ }^{\prime}$ and $\mathrm{ct}_{\mathrm{l}}{ }^{\prime}=\mathrm{c} \gamma(1-\mathrm{v} / \mathrm{c}) \mathrm{t}_{1}$. From these two equations, $\gamma=1 /\left(1-\mathrm{v}^{2} / \mathrm{c}^{2}\right)^{0.5}>0$ and $\mathrm{b}=\gamma \mathrm{v}$. Therefore, $\mathrm{x}=\left(\mathrm{x}^{\prime}+\mathrm{vt}_{1}\right) /\left(1-\mathrm{v}^{2} / \mathrm{c}^{2}\right)^{0.5}$ and $\mathrm{t}_{1}=\mathrm{x} / \mathrm{c}$ which are the exact Lorentz transformation of the special relativity for the flat space. Then, $\Delta x=\sqrt{\frac{1-\frac{v}{c}}{1+\frac{v}{c}}} \Delta x^{\prime}$ (see Figs. 7, 14 and 15) which is called as the energy transition in Figs. 7 and 15. If $\mathrm{v}<0$, then $\mathrm{c}<0$. Always $\mathrm{v} / \mathrm{c}>0$. Note that it is different from the solution of $\Delta x=\Delta x^{\prime} / \sqrt{1-\frac{v^{2}}{c^{2}}}$ which is called as the momentum transition in Fig. 15. The special and general relativity theories proposed by Einstein should be separated into the energy transition associated with the space-time shape transition of the matter and the 
momentum transition associated with the space-time location transition as shown in Figs. 7 and 15.

For the warped space, the general FLRW and quantum metrics and general space transformation of $\mathrm{X}^{\mu}=\Lambda^{\mu}{ }_{v} \mathrm{X}^{v}$ need to be used with the Einstein field equation as shown in Figs. 11 and 12. Now the time $(\mathrm{t})$ is generally defined as the proper time in the present work. Then, the proper time width in the energy transition of the special relativity in Figs. 7, 14 and 15 is $\Delta \mathrm{t}=0$ because it applied on the flat space as shown in Fig. 15. And the proper time width in the general relativity of Fig. 14 is the non-zero $\Delta \mathrm{t}$ when it is applied on the warped space in Figs. 11-14. When the energy is added to the zero curvature time-space, the zero curvature time-space is warped. Then the observed time width and observed space length between two fixed points are different from the proper time width and space length in the zero curvature time-space, respectively, because the path between two fixed points is on the curved space-time. Therefore, the special and general relativity theories for the energy transition are discussing about the relation between the energy and changes of the curvature and time-space distance. Basically, the quantum mechanics based on the shape (energy) change of the time-space is discussing about the relation between the energy and size changes of the space and time in terms of the present three-dimensional quantized space model. In other words, for the same warped space and time, the general relativity is about the curvature changes of the space and time and the quantum mechanics is about the size changes of the space and time. Relativistic effect on the size of the particle with the non-zero rest mass is shown in Fig. 7. When the particle is moving with the velocity of $\mathrm{v}$, the space size of the particle is increasing from $\Delta \mathrm{x}_{0}^{\prime}$ to $\Delta x=\sqrt{\frac{1+\frac{v}{c}}{1-\frac{v}{c}}} \Delta x /$ (see Figs. 7, 14 and 15) which is called as the energy transition in Figs. 7 and 15. Note that it is different from the solution of $\Delta x=\Delta x^{\prime} / \sqrt{1-\frac{v^{2}}{c^{2}}}$ which is called as the momentum transition in Fig. 15. The time width of the particle is decreasing from $\Delta \mathrm{t}_{0}^{\prime}$ to $\Delta \mathrm{t}=$ $\Delta \mathrm{t}_{0} /(1+\mathrm{v} / \mathrm{c})$ in Fig. 7. In Fig. 15, the energy and space-time momentum transitions of the photon, quantum space and particle are compared. The quantum space fluctuation and the photon fluctuation are the background flat space fluctuation. Because it has the zero rest mass, it does not have the time-space warped shape. Therefore, the information of the energy and space momenta is transferred to the next quantum space or photon by the background flat space fluctuation. All of the quantum spaces on the flat space have the same proper time. Therefore, the proper time difference $(\Delta t)$ of two quantum spaces is zero as shown in Fig. 15. Then, the quantum space fluctuation has the constant speed of $\mathrm{c}=\Delta \mathrm{x} / \Delta \mathrm{t}_{1}$. And the time and space momentum of the quantum fluctuation is $\mathrm{p}_{\mathrm{t}}=\mathrm{E}_{0} / \mathrm{c}=\hbar \omega / c$ and $\mathrm{p}_{\mathrm{x}}=\mathrm{E}_{0} / \mathrm{c}=\hbar k$, respectively. The energy and momenta are transferred like the wave. Therefore, this energy and momentum transitions are expressed as the wave function. If this wave-like motion is toward the $+x$ and $+t$ direction, the proper wave function is $\psi(x, t)=\frac{1}{\sqrt{\Delta x_{w} c \Delta t_{w}}} e^{\frac{i}{\hbar} P_{x} x} e^{\frac{i E_{0}}{\hbar c} c t}$ as explained in section 2.1. Here, $\mathrm{E}_{0}$ is $\Delta \mathrm{x}_{\mathrm{w}} \mathrm{c} \Delta \mathrm{t}_{\mathrm{w}}$ in Fig. 15. The quantum space fluctuation has the quantum time scale of $t_{\mathrm{q}}$ and the photon fluctuation has roughly the Planck time scale of $t_{p}$. It indicates that the increasing (flowing) of the proper time (t) and observed time $\left(\mathrm{t}_{1}\right)$ is originated from the quantum space fluctuation. The particle has the non-zero rest mass which means the warped space-time shape. This warped space-time shape is moving with the energy and space momenta when the particle is moving by changing the space location as shown in Fig. 15. The momentum transition can be solved by the point particle approximation. 
This particle is moving from one position to another position with the speed of $\mathrm{v}=\Delta \mathrm{x} / \Delta \mathrm{t}$. While it is moving, the proper time ( $t$ ) of the particle location is increasing along with the increasing of the observed time $\left(\mathrm{t}_{1}\right)$ of the particle location as shown in Fig. 15. This gives the quantum matrix of $\mathrm{c}^{2} \Delta \mathrm{t}^{2}=\mathrm{c}^{2} \Delta \mathrm{t}_{1}^{2}-\Delta \mathrm{x}^{2}$. Therefore, $\Delta t_{l}=\frac{\Delta t}{\sqrt{1-\frac{v^{2}}{c^{2}}}}$. This can explain the observed half-life of the moving particle longer than the half-life of the rest particle. In other words, if the half-life $\left(t_{1 / 2}\right)$ of the rest particle is $\mathrm{t}_{1 / 2}$, the observed half-life $\left(\mathrm{t}_{1}\right)$ of the particle moving with the speed of $\mathrm{v}$ is $t_{l}=$ $\frac{t_{1 / 2}}{\sqrt{1-\frac{v^{2}}{c^{2}}}}$. The space and time momenta are defined, in the present work, as $p_{x}=\frac{E_{0}}{c} \frac{\Delta x}{c \Delta t}=$ $\frac{E_{0}}{c} \frac{\Delta x}{c \Delta t_{l} \sqrt{1-\frac{v^{2}}{c^{2}}}}=\frac{E}{c} \frac{\Delta x}{c \Delta t_{l}}=\frac{E}{c^{2}} v$ and $p_{t}=\frac{E_{0}}{c} \frac{c \Delta t}{c \Delta t}=\frac{E_{0}}{c}$, respectively. When $\Delta \mathrm{x}_{0}=\mathrm{v} \Delta \mathrm{t}$, in Fig. 15, $\Delta x=$ $\frac{\Delta x_{0}}{\sqrt{1-\frac{v^{2}}{c^{2}}}}$ for the momentum transition. The energy transition of the moving particle is solved by giving the space-time space size to the particle as shown in Fig. 15. The $\mathrm{x}$-size of the particle is $\Delta \mathrm{x}^{\prime}$ when the $\mathrm{v}$ value is zero and $\Delta \mathrm{x}$ when it is moving with the non-zero $\mathrm{v}$ value. The relation between $\Delta \mathrm{x}^{\prime}$ and $\Delta \mathrm{x}$ can be obtained by using the special relativity for the energy transition in Fig. 14. In other words, when the particle is moving with the velocity of $\mathrm{v}$, the space size of the particle is increasing from $\Delta \mathrm{x}_{0}^{\prime}$ to $\Delta x=\sqrt{\frac{1+\frac{v}{c}}{1-\frac{v}{c}}} \Delta x^{\prime}$ for the energy transition as shown in Figs. 7, 14 and 15. If $\mathrm{v}<0$, then $\mathrm{c}<0$. Then, always $\mathrm{v} / \mathrm{c}$ is positive. Note that it is different from the solution of $\Delta x=$ $\frac{\Delta x_{0}}{\sqrt{1-\frac{v^{2}}{c^{2}}}}$ for the momentum transition in Fig. 15. The energy (E) of the moving particle is $\mathrm{E}=\Delta \mathrm{xc} \Delta \mathrm{t}_{\mathrm{w}}$ under the flat space approximation as shown as the red square in Fig. 15. It is explained in Fig. 7, too. The particle wave function can be obtained as explained in the section 2.1. It indicates that the particle motion needs to be treated separately in terms of the momentum transition and the shape (energy) transition of the particle.

\section{Quantum entanglement and quantum wave function collapse}

Quantum entanglement has never been explained successfully. In Fig. 16, one example of the quantum entanglement is shown. Two electrons marked as A and B are entangled in the singlet state $(\mid 0,0>)$. If we detect the A electron with the spin up state $(\mid 1 / 2,1 / 2>)$, the $\mathrm{B}$ electron should have the spin down state $(\mid 1 / 2,-1 / 2>)$ regardless of the distance between two electrons. Also, if we detect the A electron with the spin down state $(\mid 1 / 2,-1 / 2>)$, the B electron should have the spin up state $(\mid 1 / 2,1 / 2>)$ regardless of the distance between two electrons. This is called as the quantum entanglement. In the present work, the quantum entanglement is explained by using the quantum base. 
Quantum entanglement between two elementary fermions is not the interaction caused by the exchange of a boson but means the connection of two elementary fermions. In other words, the A electron with the spin up state $(\mid 1 / 2,1 / 2>)$ is connected to the B electron with the spin down state $(\mid 1 / 2,-1 / 2>)$ through the quantum base and the A electron with the spin down state $(\mid 1 / 2,-1 / 2>)$ is connected to the $\mathrm{B}$ electron with the spin up state $(\mid 1 / 2,1 / 2>)$ through the quantum base as shown

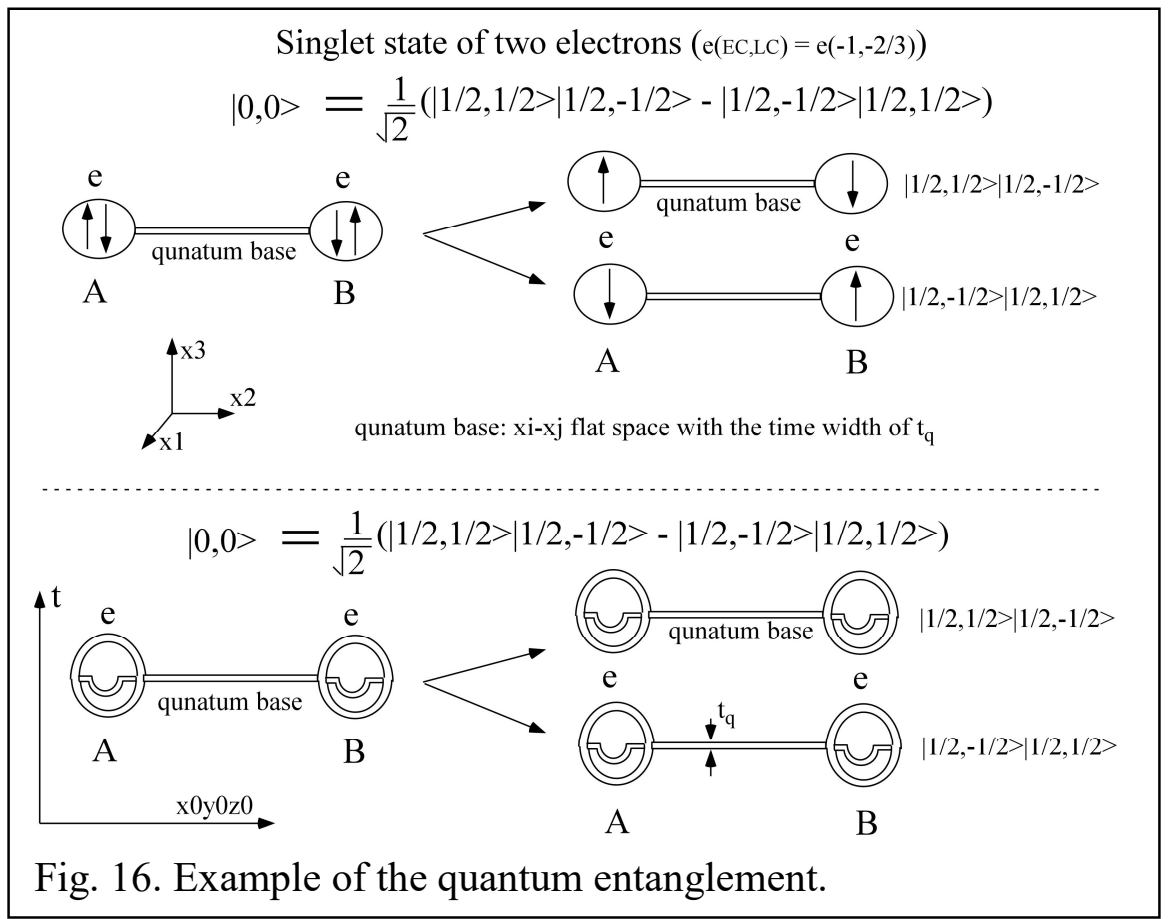

in Fig. 16. The electrons are the xi-xj (or $\mathrm{x} 2-\mathrm{x} 4$ ) particles and the quantum base between these two electrons is the $x i$-xj flat space with the time width of the quantum time $\left(t_{q}\right)$. Whenever we detect the A electron with the spin up state $(\mid 1 / 2,1 / 2>)$, the B electron should have the spin down state $(\mid 1 / 2,-1 / 2>)$ regardless of the distance between two electrons because of the quantum base. Also, whenever we detect the A electron with the spin down state $(\mid 1 / 2,-1 / 2>)$, the B electron should have the spin up state $(\mid 1 / 2,1 / 2>)$ regardless of the distance between two electrons because of the quantum base. Quantum base cannot be observed directly because the time width of the quantum time $\left(t_{q}\right)$ is much smaller than the Planck time $\left(t_{p}\right)$ which is the minimum observable time. In summary, the quantum base is introduced successfully in order to explain the quantum entanglement effects at a distance.

Now I am going to talk about the quantum wave function collapse effect and particle tunneling effect through the potential wall. The wave function, $\Psi(\mathrm{x})$, of an elementary fermion like an electron is related to the function, $\mathrm{t}(\mathrm{x})$, of the space $(\mathrm{x})$. For example, let's assume that the wave function of the electron is expressed as $\Psi(\mathrm{x})$. When we detect the electron at $\mathrm{x}=\mathrm{x}^{\prime},|\Psi(\mathrm{x})|^{2}=0$ at $x \neq x^{\prime}$ and $|\Psi(\mathrm{x})|^{2}=1$ at $x=x^{\prime}$. This is called as the quantum wave function collapse. This wave function collapse effect has never been explained successfully. In the present work, the quantum wave function collapse is explained by using the space expansion of the b-boson. And the corresponding matter within the $b$-boson [1] makes the internal vibration $(v \neq 0)$ as shown in Fig. 6. The xi-xj matter of the electron with the Planck size is oscillating within the xi-xj b-boson of the electron and the xi-xj b-boson of the electron is usually expanded along the $x i$ axis in the $\mathrm{x} 1 \times 2 \times 3$ space and the $\mathrm{xj}$ axis in the $\mathrm{x} 4 \times 5 \times 6$ space as shown in Fig. 6 . In general, the deformed 
shape $\left(|\Psi(\mathrm{x})|^{2}\right)$ of the electron b boson having the non-zero potential energy is obtained by using the Schrodinger equation of the quantum mechanics. The space-time shape of this b-boson can be expressed as $\mathrm{t}(\mathrm{x})$ and $-\mathrm{t}(\mathrm{x})$. Then, $2 \mathrm{ct}(\mathrm{x}) / \mathrm{E}$ corresponds to the quantum wave function, $|\Psi(\mathrm{x})|^{2}$, for the electron. In the present work, the wave function, $|\Psi(\mathrm{x})|^{2}$, in the quantum mechanics, is defined to be the same as the $2 \operatorname{ct}(\mathrm{x}) / \mathrm{E}$ as shown in Fig. 6.

The quantum wave function collapse of an electron is shown as an example in Fig. 6 . When the oscillation speed (v) of the $\mathrm{xi}-\mathrm{xj}$ (or $\mathrm{x} 2-\mathrm{x} 4$ ) matter is 0 which means the matter detection, an electron has the $b$ boson with the spherical space-time shape. And when the electron b-boson is moving with the velocity $\mathrm{v}$, the space width of the electron b-boson is relativistically expanded and the time width is decreased with the increasing of the electron velocity because the electron energy is conserved (see Figs. 6). Also, when the electron is detected at $\mathrm{x}=\mathrm{x}^{\prime}$, instantly the electron has the $x i-x j$ (or $x 2-x 4$ ) matter with $\mathrm{v}=0$ at $\mathrm{x}=\mathrm{x}^{\prime}$. Then the $\mathrm{xi}-\mathrm{xj}$ (or $\mathrm{x} 2-\mathrm{x} 4$ ) b-boson becomes the spherical space-time shape at $\mathrm{x}=\mathrm{x}^{\prime}$. This quantum wave function collapsing time of an electron is $\Delta \mathrm{t} \leq 2 \mathrm{r}_{\mathrm{e}} / \mathrm{c}=1.671810^{-25} \mathrm{sec}$ which is very short. The electron $\mathrm{b}$ boson radius is $\mathrm{r}_{\mathrm{e}}=2.250610^{-17}$ $\mathrm{m}$. Here $\mathrm{x}$ is $\mathrm{xi}$ (or $\mathrm{x} 2$ ) or $\mathrm{xj}$ (or $\mathrm{x} 4$ ). In order words, detecting an electron at $\mathrm{x}=\mathrm{x}^{\prime}$ means stopping the $\mathrm{xi}$-xj matter of an electron at $\mathrm{x}=\mathrm{x}^{\prime}$. Therefore, whenever we detect the electron at $\mathrm{x}=\mathrm{x}^{\prime},|\Psi(\mathrm{x})|^{2}$ $=0$ at $x \neq x^{\prime}$ and $|\Psi(\mathrm{x})|^{2}=1$ at $x=x^{\prime}$. It is the quantum wave function collapse. Therefore, the quantum wave function collapse can be generally explained by the space expansion of the electron b-boson along with the internal vibration $(\mathrm{v} \neq 0)$ of the corresponding matter within the b-boson [1].

The double slit experiment of an electron can be explained by using the space deformation of the b-boson when an electron gets through the two slits. The only option is that the b-boson should be connected between two slits so that the xi-xj matter of the electron can oscillate within the b-boson. Now the electron tunneling through the potential wall barrier is explained by using the space expansion of the electron b-boson as shown in Fig. 6. Within the wall the electron cannot exist in the viewpoint of the classical mechanics. But in the viewpoint of the quantum mechanics the electron can exist within the potential wall with the reduced probability density at $\mathrm{x}$ obtained from the space expansion of the electron b-boson. After tunneling the potential wall, the electron wave function keeps the expanded space width and reduced time width which means the reduced probability density at x. Also, in Fig. 6, it should be noted that the b-boson shape is additionally expanded into the space direction because of the energy increase by the relativistic effect when the electron is moving with a non-zero velocity. And the interference between two electron wave functions is the interference between two deformed space-time shapes of two electron b-bosons.

\section{Summary}

In summary, three-dimensional quantized spaces are newly introduced in the present work. Four three-dimensional quantized spaces with total 12 dimensions are used to explain the universes including ours as shown in Fig. 1. For the 3-dimensional quantized space, $\mathrm{E}=\mathrm{c} \Delta \mathrm{t} \Delta \mathrm{V}$ where $\Delta \mathrm{V}$ is the three-dimensional space volume. Our universe is formed with the four 3-dimensional quantized spaces. Four 3-dimensional quantized spaces can be overlapped over the same states. There is the flat mother quantized space (x0y0z0 space in Fig. 1) with the infinite time width and infinite space width. The mother quantized space cannot be warped because it has the infinite time width and infinite space width. The daughter quantized spaces $(x 1 \times 2 \times 3, x 4 \times 5 \times 6$ and $\times 7 \times 8 \times 9$ spaces in Fig. 
1) with the finite time width and finite space width can be warped on the flat mother quantized space. Therefore, if one daughter quantized space is warped, other daughter quantized spaces are warped, too. The warped space is defined with the charge and rest mass. The negative warping of the quantized space is defined as the negative charge which means the matters. The birth of our positive energy matter universe is justified from the production of the positive and negative energy warped space pair. This birth is the beginning of the physical universe with the big bang. The warped space is the unstable space with the excited warping energy and the flat space is the stable space with the minimum energy.

It indicates that when it is based on the reference energy of $E_{0}$, the well-known Schrodinger equation of

$E_{s} \psi_{s}(x)=\left(E-E_{0}\right) \psi_{s}(x)=H \psi_{s}(x)=\left(\frac{p_{x}^{2}}{2 m}+V(x)\right) \psi_{s}(x)$ can be used instead of the original Schrodinger equation of $E \psi(x)=H \psi(x)=\left(E_{0}+\frac{p_{x}^{2}}{2 m}+V(x)\right) \psi(x)$. And based on the reference energy of $E_{0}, E_{\mathrm{s}} \psi_{\mathrm{s}}(\mathrm{t})=-i \hbar \frac{d}{d t} \psi_{\mathrm{s}}(\mathrm{t})$ and $\psi_{s}(t)=\frac{1}{\sqrt{c \Delta t_{s}}} e^{\frac{i E_{s}}{\hbar c} c t}$ can be used instead of the original equations of $\mathrm{E} \psi(\mathrm{t})=-i \hbar \frac{d}{d t} \psi(\mathrm{t})$ and $\psi(t)=\frac{1}{\sqrt{c \Delta t}} e^{\frac{i E}{\hbar c} c t}$ obtained by the flat space approximation. Then , $\psi(x)=\psi_{s}(x)$ and

$\psi(t)=\frac{1}{\sqrt{c \Delta t}} e^{\frac{i E}{\hbar c} c t}=\frac{\sqrt{E-E_{0}}}{\sqrt{E}} e^{\frac{i E_{0}}{\hbar c} c t} \psi_{s}(t)$.

The quantum entanglement and quantum wave function collapse are explained additionally.

Now the time $(\mathrm{t})$ is generally defined as the proper time in the present work. Then, the proper time width in the energy transition of the special relativity in Figs. 7, 14 and 15 is $\Delta t=0$ because it applied on the flat space as shown in Fig. 15. And the proper time width in the general relativity of Fig. 14 is the non-zero $\Delta \mathrm{t}$ when it is applied on the warped space in Figs. 11-14. When the energy is added to the zero curvature time-space, the zero curvature time-space is warped. Then the observed time width and observed space length between two fixed points are different from the proper time width and space length in the zero curvature time-space, respectively, because the path between two fixed points is on the curved space-time. Therefore, the special and general relativity theories for the energy transition are discussing about the relation between the energy and changes of the curvature and time-space distance. Basically, the quantum mechanics based on the shape (energy) change of the time-space is discussing about the relation between the energy and size changes of the space and time in terms of the present three-dimensional quantized space model. In other words, for the same warped space and time, the general relativity is about the curvature changes of the space and time and the quantum mechanics is about the size changes of the space and time. Then, the quantum mechanics and the general relativity theory are about the 4-dimensional space-time volume and the 4-dimensional space-time distance, respectively, as shown in Table 1. 
The present new three-dimensional quantized space model is explained in the article [1] and its revised versions on the three-dimensional quantized spaces, elementary particles and quantum mechanics.

References

[1]. J.K. Hwang, www.researchgate.net ; DOI:10.13140/RG.2.1.2388.4561/1

and its revised versions; https://www.researchgate.net/profile/J Hwang2 